\title{
Evaluation Method for Determining Management Priorities for Special Case Waste
}

D. E. Kudera

C. E. Wickland

August 1990
RECEIVED

AUG 221998

OSTI

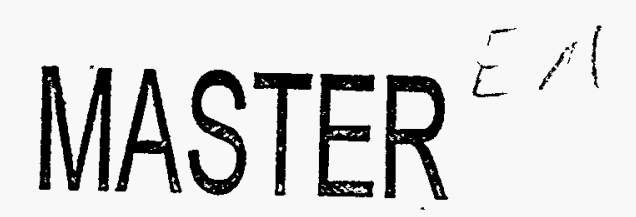




\author{
D. E. Kudera \\ C. E. Wickland
}

Published August 1990

Idaho National Engineering Laboratory

EG\&G Idaho, Inc.

Idaho Falls, Idaho 83415

Prepared for the

U.S. Department of Energy

Idaho Operation Office

Under DOE Contract No. DE-AC07-76ID01570 


\section{DISCLAIMER}

This report was prepared as an account of work sponsored by an agency of the United States Government. Neither the United States Government nor any agency thereof, nor any of their employees, makes any warranty, express or implied, or assumes any legal liability or responsibility for the accuracy, completeness, or usefulness of any information, apparatus, product, or process disclosed, or represents that its use would not infringe privately owned rights. Reference herein to any specific commercial product, process, or service by trade name, trademark, manufacturer, or otherwise does not necessarily constitute or imply its endorsement, recommendation, or favoring by the United States Government or any agency thereof. The views and opinions of authors expressed herein do not necessarily state or reflect those of the United States Government or any agency thereof. 


\section{DISCLAIMER}

Portions of this document may be illegible in electronic image products. Images are produced from the best available original document. 


\section{CONTENTS}

ACRONYMS ............................ . . . v

INTRODUCTION . . . . . . . . . . . . . . . . . . . . . . . 1

DECISION TREE ANALYSIS . . . . . . . . . . . . . . . . . 3

TREATMENT/STORAGE/DISPOSAL AT SITES HOLDING SPECIAL CASE WASTE • . . . 8 SPECIAL CASE WASTE DATA AND PRIORITY EVALUATION . . . . . . . . . . . 13

OVERALL PRIORITY EVALUATION RANKING . . . . . . . . . . . . . . . 28 RESULTS . . . . . . . . . . . . . . . . . . . 32 CONCLUSIONS . . . . . . . . . . . . . . . . . . . . . 33 APPENDIX A - SPECIAL CASE WASTE TREATMENT AND DISPOSAL STRATEGY DECISION TREE ..................... . . . . . .

\section{TABLES}

1. Hanford Waste management capabilities . . . . . . . . . . . 8

2. Idaho National Engineering Laboratory waste management capabilities . 8

3. Los Alamos National Laboratory waste management capabilities . . . . 9

4. Lawrence Berkeley Laboratory waste management capabilities . . . . 9

5. Lawrence Livermore National Laboratory waste management capabilities ......................... 9

6. Mound Laboratory waste management capabilities . . . . . . . . . 10

7. Nevada Test Site waste management capabilities . . . . . . . . 10

8. Oak Ridge Gaseous Diffusion Plant waste management capabilities . . . 10

9. Oak Ridge National Laboratory waste management capabilities . . . . 11

10. Oak Ridge Y-12 Plant waste management capabilities . . . . . . . . 11

11. Paducah Gaseous Diffusion Plant waste management capabilities . . . 11

12. Portsmouth Gaseous Diffusion Plant waste management capabilities . . 11

13. Rocky Flats Plant waste management capabilities . . . . . . . . 12 
14. Sandia National Laboratory-Albuquerque waste management

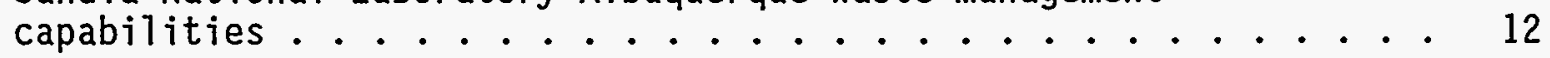

15. Savannah River waste management capabilities . . . . . . . 12

16A. Albuquerque special case waste information ......... 14

16B. Albuquerque Field Office special case waste priority evaluation . . 14

17A. Chicago special. case waste information ......... 15

17B. Chicago Field office special case waste priority evaluation . . . 15

18A. Idaho special case waste information . . . . . . . 16

18B. Idaho Field Office special case waste priority evaluation . . . . 16

19A. Naval Reactors special case waste information . . . . . . . 17

19B. Naval Reactors special case waste priority evaluation . . . . . 17

20A. Nevada special case waste information ........... 18

20B. Nevada special case waste priority evaluation ......... 18

21A. Oak Ridge special case waste information . . . . . . . 19

21B. Oak Ridge Field Office special case waste priority evaluation . . 20

22A. Richland special case waste information .......... 21

22B. Richland Field Office special case waste priority evaluation . . 22

23A. Rocky Flats special case waste information ......... 23

23B. Rocky Flats special case waste priority evaluation ....... 23

24A. San Francisco special case waste information ........ 24

24B. San Francisco Field Office special case waste priority evaluation . 24

25A. Savannah River special case waste information ........ 25

25B. Savannah River special case waste priority evaluation . . . . . 25

26. SCW potëntial resolution key ............... 29

27. Highest ranked SCW items for continued storage (a11 field offices) . 30

28. Highest ranked SCW items for implementing treatment (all field offices . . . . . . . . . . . . . . 31 


\section{ACRONYMS}

CCSA Continued Current Storage Activities

$\mathrm{CH} \quad$ Contact Handled

CIF Consolidated Incineration Facility

DOE U.S. Department of Energy

DP Defense Programs

DRS Data Record Sheet

DTA Decision Tree Analysis

DWPF Defense Waste Processing Facility

DWTF Defense Waste Treatment Facility

EDL Economic Discard Limit

EU Enriched Uranium

FMPC Feed Material Production Center

GCD Greater Confinement Disposal

$\mathrm{HAZ} \quad$ Hazardous

HLW High Level Waste

HQ Headquarters

IBAT Implementation of the Best Available Treatment

LAMPRE LOS ATamos Molten Plutonium Reactor Experiment

LANL LOS Alamos National Laboratory

LBL Lawrence Berkeley Laboratory

LLMW Low-Level Mixed Waste

LLNL Lawrence Livermore National Laboratory

LLW Low-Level Waste

MAP Mixed Activation Products

MFP Mixed Fission Products

MPPF Multi-Purpose Processing Facility

MW Mixed Waste

PA Performance Assessment

PAL Performance Assessment Limiting

PCP Payload Compliance Plan

PE . Priority Evaluation

PR Priority Rating

RCRA Resource Conservation and Recovery Act

RH Remote Handled

SCW Special Case Waste

SLB Shallow Land Burial

SPAR Specific Performance Assessment Required

SWEPP Stored Waste Examination Pilot PTant

TRU Transuranic

TRUPACT Transuranic Package Transporter

TSCA Toxic Substances Control Act

TSP Radioactive Waste Technical Support Program

TWF Transuranic Waste Facility

WAC Waste Acceptance Criteria

WG Weapons Grade

WIPP Waste Isolation Pilot Plant

WRAP Waste Receiving and Packaging Facility 


\section{EVALUATION METHOD FOR DETERMINING MANAGEMENT PRIORITIES FOR SPECIAL CASE WASTE}

\section{INTRODUCTION}

The U.S. Department of Energy (DOE) Radioactive Waste Technical Support Program (TSP) began the Special Case Waste (SCW) Inventory and Characterization Project in April 1989. The collection of data has been completed and a final draft report, Department of Energy Special Case Radioactive Waste Inventory and Characterization Data Report (DOE/LLW-96), was submitted in May 1990. A second final draft report, Supplemental Data Report to the Department of Energy Special Case Radioactive Waste Inventory and Characterization Data Report (DOE/LLW-95), containing additional and more detailed data and graphical presentations, was completed in July 1990. These two reports contain details on the special case waste categories and summaries of the total volumes and curies associated with each category of waste. It is anticipated that some version or combination of these two reports will be included in the final version of this report, which will describe an evaluation method for determining management priorities for special case waste. Preliminary analysis of the inventory data indicates that approximately $1,000,000 \mathrm{~m}^{3}$ of special case waste exist in the DOE system with possible insufficient treatment/storage/disposal capability or capacity. To help DOE prioritize the actions required to manage this large volume of special case waste, an evaluation method is required.

The purpose of this report is to describe a decisionmaking process for managing special case waste. This process is developed from a risk, needs, and cost based evaluation that results in a numerical rating for each waste item for continuing storage or implementing treatment. The priority evaluation described in this report will enable DOE to reach informed decisions about whether to act to implement the best available treatment or delay action and continue current storage activities. 
There are two basic activities that must be performed to accomplish this task. The first activity is to apply a Decision Tree Analysis (DTA) to identify special case waste that warrants further evaluation. The second activity is to perform a Priority Evaluation (PE) on those wastes identified in the Decision Tree Analysis as being a continuing problem. Problem wastes consist of wastes with limited or no planned disposal alternatives; therefore, they require treatment or some other action. A waste is considered a problem for the following reasons:

- Risk or perceived risk to workers, public or the environment

- Lack of treatment availability

- Cost to develop treatment technology

- Failure to meet regulatory requirements

- Lack of disposal facilities.

A priority evaluation is needed to identify special case waste that warrants a higher priority for implementing action. The priority evaluation uses six parameters to numerically prioritize the possible actions for special case waste. The priority evaluation will enable DOE to make the most effective and efficient decisions regarding which special case wastes require immediate action and which can continue to be safely stored. These decisions are based on risk, needs, and cost. Summary tables in this report present a ranking of the priority evaluations and show the special case waste items that could continue to be stored and the special case waste items that should receive the best available treatment. 


\section{DECISION TREE ANALYSIS}

A Decision Tree Analysis is performed for most Data Record Sheets (DRSs) submitted for the DOE special case waste project. No Decision Tree Analysis, priority evaluation, or potential resolution will be performed for loan/lease materials (DOE-titled, but held by NRC licensees) and sealed sources (encapsulated material whose main purpose is to generate known amounts of radiation). These categories involve over 6,000 items with similar management problems. A potential resolution for loan/lease material and sealed sources can be arrived at without doing an individual priority evaluation for each item. The potential resolutions for these special case waste categories are not part of this report. All other data record sheets for the other special case waste categories have been evaluated in this report.

The Special Case Waste Treatment and Disposal Strategy Decision Tree, in Appendix A, graphically presents the logical steps in the Decision Tree Analysis determination of the waste management methods that must be applied to obtain final disposal of the special case waste. Problem wastes, designated $B$, are identified at various locations on the flow chart. These wastes require a priority evaluation and result from the following types of problems and potential resolutions:

Problem Description

Not transportable for offsite disposal No treatment available (on- or offsite) Not transportable for offsite treatment Offsite treatment still leaves problems No disposal site
Resolution

Onsite treatment

Develop treatment method

Onsite treatment

Better planning

Develop disposal capability

- In addition to identifying problem wastes, the decision tree also identifies two actions required for some special case waste: characterization and onsite treatment. Waste that must be characterized or treated onsite also requires a priority evaluation. The characterization methods, including sampling of the waste, must be done onsite; otherwise, it cannot be determined if the uncharacterized waste will meet the transportation criteria. If immediate onsite treatment of the waste is available to render it a nonspecial 
case, the waste is probably not a special case waste. However, future onsite treatment facilities may only be $\mathrm{planned;}$ therefore, continued storage of the special case waste is required.

Review of this Decision Tree Analysis shows that each site that has problem waste must have onsite facilities to characterize and treat that waste. In some cases the treatment may be simply repackaging to meet transportation criteria for offsite shipment for disposal or further treatment. However, it must be ensured that any offsite treatment will result in eventual disposal of the waste. 


\section{PRIORITY EVALUATION}

Based on the Decision Tree Analysis all special case waste that requires treatment will receive a priority evaluation. An outline for performing the priority evaluation is shown in Figure 1.

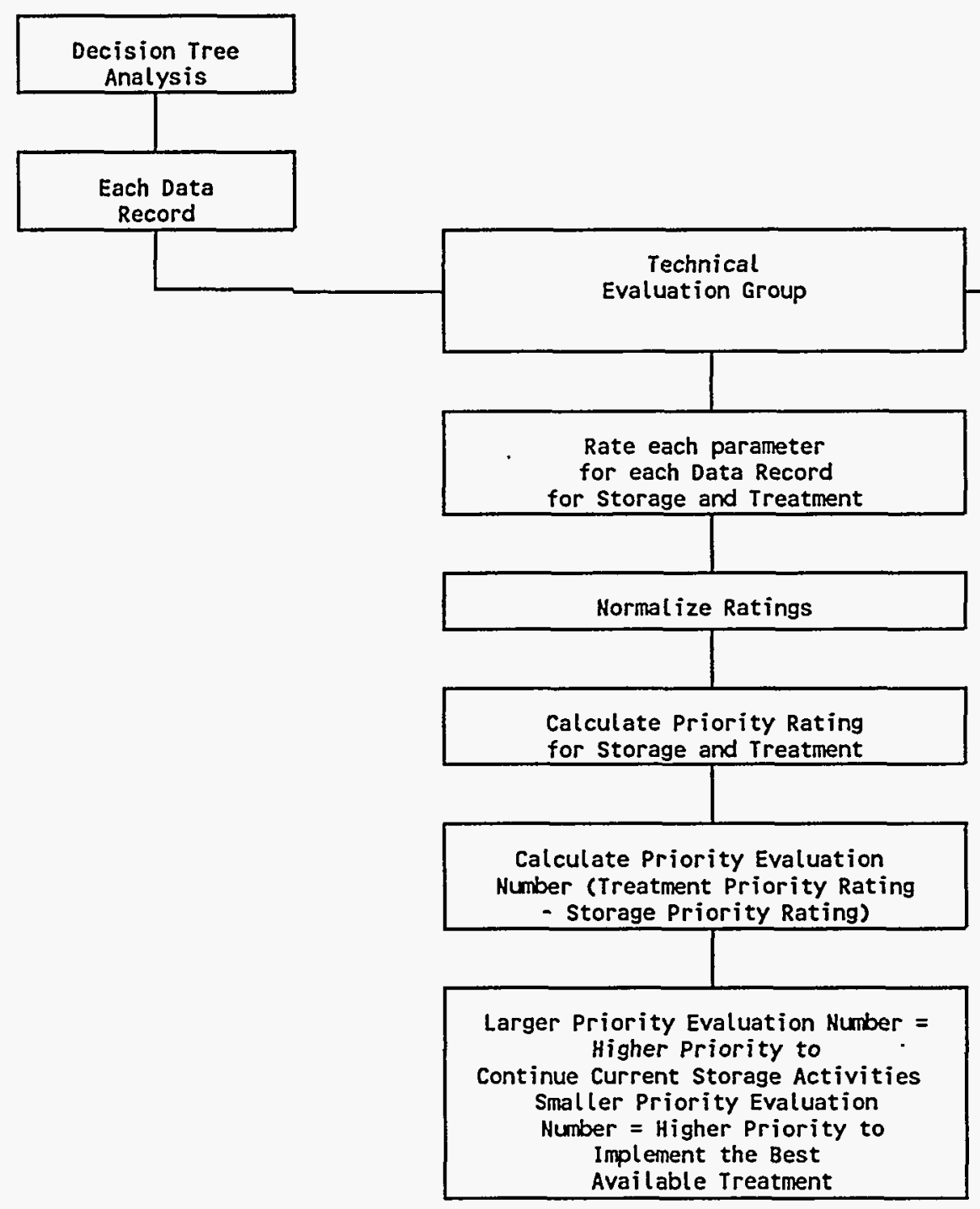

Figure 1. Guideline for priority evaluation of special case waste for management decisions. 
The priority evaluation provides a method for DOE to make the most effective and reasonable decision regarding which special case wastes require immediate action and which can continue to be stored safely.

For this report, the priority evaluation is performed by the TSP. However, improvement to the priority evaluation may be achieved by a technical evaluation group of recognized experts from the various field offices in the DOE complex. These individuals should be site representatives that are acknowledged experts in treatment, storage, and disposal capabilities. Individual evaluations could then be collected and normalized by personnel designated by DOE-Headquarters.

Six parameters are evaluated for each priority evaluation: estimated risk (environmental and health), (2) perceived risk (institutional and public), (3) regulatory requirements, (4) availability of storage or treatment, (5) feasibility of storage or treatment, and (6) estimated cost. These parameters are weighted by relative importance. The most important is rated 10, and the least important could be rated as low as 1 . The weighting factors applied to these parameters must be given careful consideration because they greatly influence the final result. Suggested weighting factors that are used in this evaluation are as follows:

- Environmental/health estimated risk - 10

- Institutional/public perceived risk- 5

- Regulatory compliance - 9

- Availability of storage/treatment - 8

- Feasibility of storage/treatment - 8

- Estimated cost - 7

The evaluation is performed for continuing storage activities and for implementing the best available treatment to convert the special case waste to a non-special. case waste. The evaluation consists of rating the risk, needs and cost of continuing storage and implementing treatment for each special case waste item. Rating guidance for continuing storage and implementing treatment is as follows:

- Estimated risk (environmental and health)

Low risk - 1

Medium risk - 5

High risk - 10

- Perceived risk (institutional and public)

Low - 1

Medium - 5

High - 10

- Regulatory compliance

High compliance, minimal regulatory concerns (OK) - I

Unknown compliance, hazardous constituents unknown (?) - 5

High noncompliance, violation of agreements (problem) - 10 
- Availability

Yes - 1

No -10

- Feasibility

No problem (OK) - 1

Unknown (?) - 5

Space or capability/capacity problem - 10

- Estimated cost

Low - 1

Medium - 5

High - 10 .

Multiplication of the rating by the weighting factor for each parameter and then summing the numbers for each parameter yields a numerical expression of the overall problem, which is called the priority rating (PR). A priority rating is calculated in this manner for continued storage and implementing treatment for each special case waste item.

The priority evaluation is then determined by subtracting the priority rating for storage from the priority rating for treatment for each data record sheet. The difference is the priority evaluation, which can be a positive or negative number. The larger the priority evaluation number, the higher the priority to continue current storage activities. The smaller the priority evaluation number, the higher the priority to act to implement the best available treatment. However, continued storage is not the final solution because the environmental and health risk wi17 increase over time, and the storage capacity may be 7 imited. Some form of treatment will eventually be required for most of the special case wastes that require a priority evaluation because permanent disposal is the ultimate goal.

The Decision Tree Analysis/priority evaluation process will direct which special case wastes warrant immediate treatment. Because this is strictly a tool to aid in decisionmaking, other overriding considerations may influence any final decisions. 


\section{TREATMENT/STORAGE/DISPOSAL AT SITES HOLDING SPECIAL CASE WASTE}

To perform a priority evaluation, one must be informed of the treatment, storage, and disposal capabilities and limitations of each site. A preliminary evaluation of these capabilities and limitations was made by reviewing the 1989 site Waste Management Implementation Plans. The pertinent information obtained from this review is listed in Tables 1 through 15 . This information was used as an aid to decide how much of a problem continued storage or treatment of the special case waste might be. Changes to this information may influence the results of the priority evaluation. This information should be updated before any future priority evaluations are conducted.

Some information that may influence the priority evaluation, such as treatment capacity, was not always available. To conduct a more accurate priority evaluation, the evaluation must be redone using recognized experts from the various field offices in the DOE complex to provide a consensus evaluation.

Table 1. Hanford Haste management capabilities

\begin{tabular}{|c|c|c|c|c|}
\hline 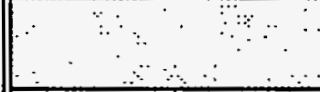 & $\begin{array}{l}\text { Un } \\
\text { High-Level Waste }\end{array}$ & Transuraniculaste & Loh-tevel Haste & $\begin{array}{l}\text { Hixed } \\
\text { Haste. }\end{array}$ \\
\hline TREATHENT' & Vitrification (99) & $\begin{array}{l}\text { Vitrification (99) } \\
\text { Segregation }(96) \\
\text { Repackaging }(96) \\
\text { (URAP-96 \& } 99)^{\text {a }}\end{array}$ & $\begin{array}{l}\text { Cementation } \\
\text { (WRAP-96 \& 99) }\end{array}$ & $\begin{array}{l}\text { None } \\
\text { WRAP }\end{array}$ \\
\hline STORAGE & $\begin{array}{l}\text { Water Basin } \\
\text { Storage Tanks }\end{array}$ & $\begin{array}{l}\text { Temporary } \\
\text { ( } 25 \text { year) }\end{array}$ & $\begin{array}{l}\text { Temporary } \\
\text { ( } 25 \text { year) }\end{array}$ & Temporary \\
\hline olsposni & Hone & None & $\begin{array}{l}\text { Shallow Land } \\
\text { Burial Concrete } \\
\text { Vaults }\end{array}$ & None \\
\hline
\end{tabular}

a. HRAP $=$ Waste Receiving and Packaging Facility

Table 2. Idaho National Engineering Laboratory waste management capabilities

\begin{tabular}{|c|c|c|c|c|}
\hline \begin{tabular}{cc} 
& $\ddots$ \\
$\therefore$ & $\ddots$ \\
\hdashline
\end{tabular} & High-Level Uaste & $\begin{array}{c}\because \cdots \\
\text { Transuranic Haste } \\
\end{array}$ & $\begin{array}{r}\text { Low-Level: } \\
\text { Naste }\end{array}$ & $\begin{array}{c}\text { Mixed Waste } \\
\text { Mist }\end{array}$ \\
\hline 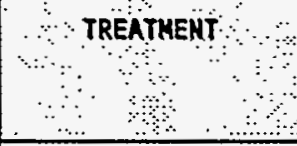 & $\begin{array}{l}\text { Solidification } \\
\text { Evaporation }\end{array}$ & $\begin{array}{l}\text { Shredding (93) } \\
\text { Compaction (93) } \\
\text { Repackaging (93) } \\
\text { Incineration (92) } \\
\end{array}$ & $\begin{array}{l}\text { Incineration } \\
\text { Compaction } \\
\text { Metal Sizing }\end{array}$ & None \\
\hline $\begin{array}{c}\text { STORAGE } \\
\therefore \\
0\end{array}$ & $\begin{array}{c}\text { Underground Tanks } \\
\text { Concrete Vaults } \\
\text { Hater Pools } \\
\text { Dry Casks }\end{array}$ & $\begin{array}{l}\text { Covered Pads } \\
\text { Buildings }\end{array}$ & Not Applicable & $\begin{array}{l}\text { Covered Pads } \\
\text { Buildings }\end{array}$ \\
\hline DISPOSAL & None & None & SLB & None \\
\hline
\end{tabular}


Table 3. Los Alamos National Laboratory waste management capabilities

\begin{tabular}{|c|c|c|c|c|}
\hline & High-Level Waste & $\begin{array}{l}\text { Transuranic } \\
\text { Waste }\end{array}$ & Low-level Waste & $\because \quad$ Mixed Waste $\because$ \\
\hline TREATMEHT & Incineration & $\begin{array}{l}\text { Precipitation } \\
\text { Ion Exchange } \\
\text { Size Reduction } \\
\text { Incineration } \\
\text { Repackaging } \\
\end{array}$ & $\begin{array}{l}\text { Incineration (91) } \\
\text { Compaction } \\
\text { Precipitation } \\
\text { Ion Exchange }\end{array}$ & Incineration \\
\hline $\begin{array}{r}\text { STORAGE: } \\
\vdots \\
\because \quad \because\end{array}$ & Modified Shafts & $\begin{array}{l}\text { Asphalt Pads } \\
\text { Modified Pits } \\
\text { Concrete Casks }\end{array}$ & Hot Applicable & $\begin{array}{l}\text { Earthen Bermed Areas } \\
\text { Resource Conservation } \\
\text { and Recovery Act } \\
\text { Storage Pads ( } 91 \text { ) }\end{array}$ \\
\hline DISPOSAL & None & None & $\begin{array}{l}\text { Large Pits } \\
\text { Greater Conf inement } \\
\text { Disposal } \\
\text { Augered Shafts }\end{array}$ & $\begin{array}{l}\text { Resource Conservation and } \\
\text { Recover Act Landfill ( } 92)\end{array}$ \\
\hline
\end{tabular}

Table 4. Lawrence Berkeley Laboratory waste management capabilities

\begin{tabular}{|c|c|c|c|c|}
\hline & $\begin{array}{c}\text { High-Level } \\
\text { Waste }\end{array}$ & Transurmic Waste & Low tevel laste & Wixed Waste \\
\hline $\begin{array}{l}\text { TREATHEMT' } \\
.\end{array}$ & Hone & Evaporation & $\begin{array}{l}\text { Crushing } \\
\text { Compaction } \\
\text { Precipitation } \\
\text { Solidification } \\
\text { Segregation }\end{array}$ & Compaction \\
\hline STORAGE & None & Temporary & Temporary & Temporary \\
\hline DISPOSAL & None & None & None & None \\
\hline
\end{tabular}

Table 5. Lawrence Livermore National Laboratory waste management capabilities

\begin{tabular}{|c|c|c|c|c|}
\hline & HLU. & $\begin{array}{c}\text { Transuranic } \\
\text { Waste }\end{array}$ & $\therefore$ Lou-Level Naste $\quad \therefore$ औl & 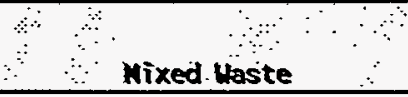 \\
\hline TREATHENT & None & $\begin{array}{l}\text { Solidification } \\
\text { Size Reduction }\end{array}$ & $\begin{array}{c}\text { Radioactive and Hazardous } \\
\text { Haste Treatment Facility }\end{array}$ & $\begin{array}{c}\text { Radioactive and Hazardous } \\
\text { Haste Treatment Facility }\end{array}$ \\
\hline STORAGE & None & Temporary & Temporary & Temporary \\
\hline DISPOSAL & None & None & None & None \\
\hline
\end{tabular}


Table 6. Mound Laboratory waste management capabilities

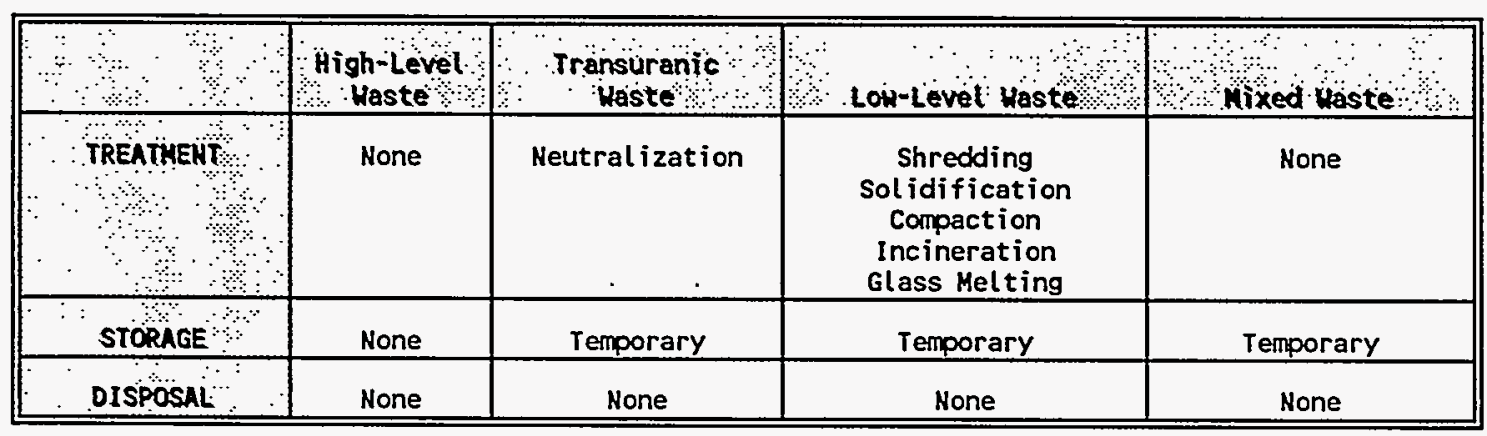

Table 7. Nevada Test site waste management capabilities

\begin{tabular}{|c|c|c|c|c|}
\hline औ & $\begin{array}{l}\text { High-Level } \\
\text { - Waste }\end{array}$ & $\begin{array}{l}\text { Tronsuranic } \\
\text { Uaste }\end{array}$ & की & Howixed Waste \\
\hline 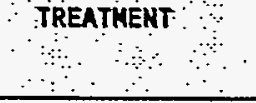 & None & None & $\begin{array}{c}\text { Solar Evaporation } \\
\text { Solidification } \\
\text { Compaction }\end{array}$ & None \\
\hline STORAGE & None & Temporary & Not Applicable & Temporary \\
\hline \begin{tabular}{ccc} 
DISPOSAL & $\ddots$ \\
& & $\ddots$ \\
\hdashline & $\ddots$ & $\ddots$ \\
\hdashline & $\ddots$ & $\ddots$ \\
\hdashline
\end{tabular} & None & None & $\begin{array}{c}\text { Greater Conf inement } \\
\text { Disposal } \\
\text { Shallow Land } \\
\text { Burial } \\
\text { Classified }\end{array}$ & Interim Status \\
\hline
\end{tabular}

Table 8. Oak Ridge Gaseous Diffusion Plant waste management capabilities

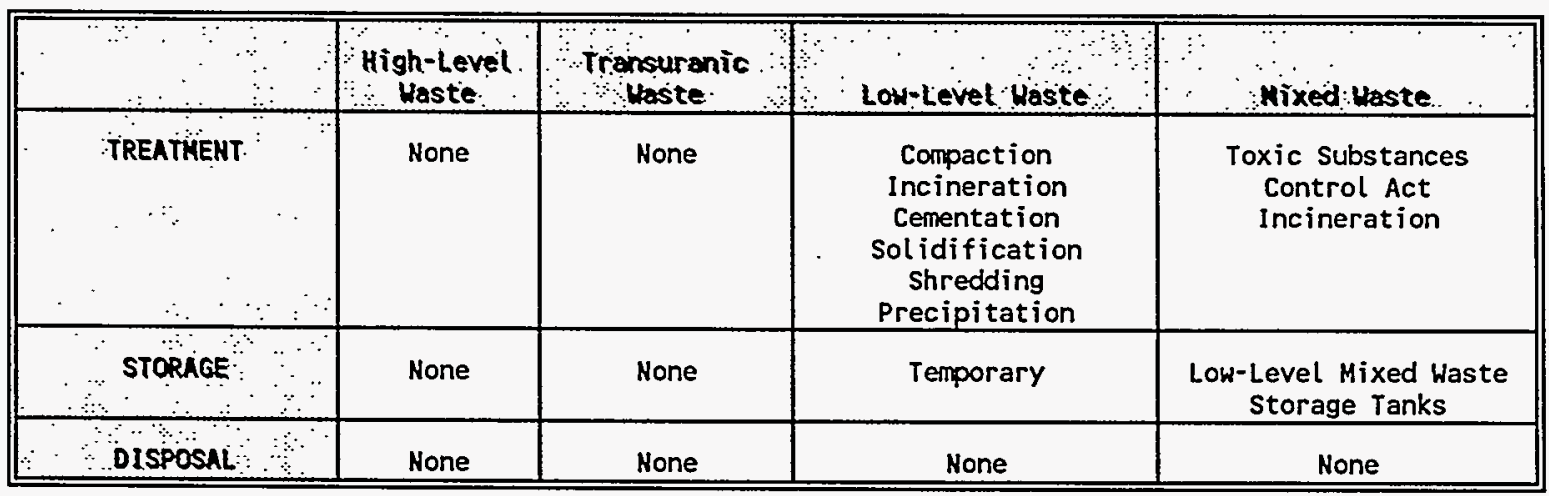


Table 9. Oak Ridge National Laboratory waste management capabilities

\begin{tabular}{|c|c|c|c|c|}
\hline . & $\begin{array}{c}\text { High-Level } \\
\text { Waste }\end{array}$ & $\begin{array}{c}\text { Transuranic } \\
\text { Uaste }\end{array}$ & Lov-Level Waste & Mixed Waste \\
\hline TREATMEHT & None & $\begin{array}{c}\text { Compaction } \\
\text { Shredding } \\
\text { Size Reduction }\end{array}$ & $\begin{array}{l}\text { Compaction } \\
\text { Cementation }\end{array}$ & None \\
\hline STORAGE & None & Temporary & Temporary & $\begin{array}{c}\text { Temporary } \\
\text { (Submitted Part B) }\end{array}$ \\
\hline DISPOSAL & None & None & $\begin{array}{c}\text { Greater Confinement } \\
\text { Disposal } \\
\text { Above Grade Tumulus }\end{array}$ & None \\
\hline
\end{tabular}

Table 10. Oak Ridge $Y-12$ Plant waste management capabilities

\begin{tabular}{|c|c|c|c|c|}
\hline & $\begin{array}{c}\text { High-Level } \\
\text { Waste }\end{array}$ & $\begin{array}{c}\text { Transuranic } \\
\text { Naste }\end{array}$ & Lou-leviel Waste & Mixed Waste \\
\hline TREATKEHT & None & None & $\begin{array}{c}\text { Production Waste } \\
\text { Treatment Facility (93) } \\
\text { Compaction } \\
\text { Supercompaction }\end{array}$ & $\begin{array}{l}\text { Production Waste } \\
\text { Treatment Facility (93) }\end{array}$ \\
\hline STCRMGE & None & None & $\begin{array}{c}\text { Classified } \\
\text { Concrete Vaults } \\
\end{array}$ & $\begin{array}{c}\text { Classified } \\
\text { Concrete Vaults }\end{array}$ \\
\hline DISPOSAL & None & None & Shallow Land Burial & None \\
\hline
\end{tabular}

Table 11. Paducah Gaseous Diffusion Plant waste management capabilities

\begin{tabular}{||c|c|c|c|c||}
\hline & $\begin{array}{c}\text { High-Level } \\
\text { Waste }\end{array}$ & $\begin{array}{c}\text { Transuranic } \\
\text { Uaste }\end{array}$ & Lon-Level Waste & Mixed Haste \\
\hline TREATHENT & None & Cementation & Precipitation & None \\
\hline STORAGE $\because$ & None & Temporary & $\begin{array}{c}\text { Temporary } \\
\text { Storage Tanks }\end{array}$ & Temporary \\
\hline DISPOSAL $:$ & None & None & None & None \\
\hline
\end{tabular}

Table 12. Portsmouth Gaseous Diffusion Plant waste management capabilities

\begin{tabular}{|c|c|c|c|c|}
\hline & $\begin{array}{c}\text { High-Level } \\
\text { Waste }\end{array}$ & $\begin{array}{c}\text { Transuranic } \\
\text { Uaste }\end{array}$ & Lou-Level Waste & Nixed Uaste \\
\hline TREATMERT & None & None & None & None \\
\hline STORAGE & None & Temporary & Temporary & None \\
\hline DISPOSAL & None & None & $\begin{array}{c}\text { Shal low Land Burial } \\
\text { Unt il 1990 } \\
\text { New Facilities (96) }\end{array}$ & \\
\hline
\end{tabular}


Table 13. Rocky Flats Plant waste management capabilities

\begin{tabular}{|c|c|c|c|c|}
\hline $\begin{array}{llll}\because & \ddots\end{array}$ & $\begin{array}{l}\text { High-Level } \\
\text { Waste: }\end{array}$ & Transuranic Uaste & Loul- Kevel Vaste & Nixed Waste \\
\hline 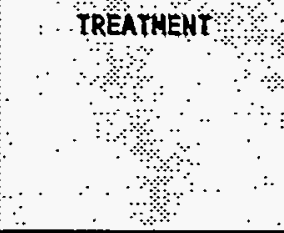 & Mone & $\begin{array}{l}\text { Size Reduction } \\
\text { Compaction } \\
\text { Precipitation } \\
\text { Imobilization } \\
\text { Solidification } \\
\text { Supercompaction } \\
\text { Microwave Helting }\end{array}$ & $\begin{array}{l}\text { Compaction } \\
\text { Immobilization } \\
\text { Cementation }\end{array}$ & None \\
\hline STORAGE & None & Temporary & None & Temporary \\
\hline oisposal: & None & None & None & None \\
\hline
\end{tabular}

Table 14. Sandia National Laboratory-Albuquerque waste management capabilities

\begin{tabular}{|c|c|c|c|c|}
\hline 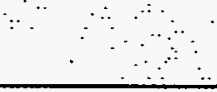 & $\begin{array}{l}\text { High Level } \\
\text { Waste }\end{array}$ & $\begin{array}{c}\text { Transuraric } \\
\text { Haste }\end{array}$ & Low-Level Naste & $\begin{array}{c}\therefore \therefore \\
\text { Nixed Waste }\end{array}$ \\
\hline TREATMENT $\because$ & None & None & $\begin{array}{c}\text { Compaction } \\
\text { Solidification } \\
\end{array}$ & $\begin{array}{c}\text { Compaction } \\
\text { Solidification }\end{array}$ \\
\hline STORAGE & None & None & Temporary & Temporary \\
\hline DISPOSAL & None & None & None & None \\
\hline
\end{tabular}

Table 15. Savannah River waste management capabilities

\begin{tabular}{|c|c|c|c|c|}
\hline$\because \because \because$ & Kigh-Level Haste & Transuranic Waste & Kow-level vaste & Hixed Waste \\
\hline 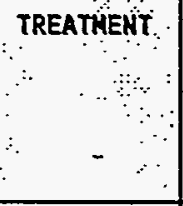 & $\begin{array}{c}\text { Evaporation } \\
\text { Filtration } \\
\text { Borosilicate Glass } \\
\text { Defense Waste } \\
\text { Processing Facility } \\
\text { (92) }\end{array}$ & $\begin{array}{l}\text { Transuranic Waste } \\
\text { Facility (90) }\end{array}$ & $\begin{array}{l}\text { Compaction } \\
\text { Filtration } \\
\text { Organic Removal } \\
\text { Reverse Osmosis } \\
\text { Ion Exchange } \\
\text { Incineration }\end{array}$ & $\begin{array}{l}\text { Consolidated } \\
\text { Incineration } \\
\text { Facility (92) }\end{array}$ \\
\hline STORAGE: & $\begin{array}{l}\text { Storage Tanks } \\
\text { Canister Storage }\end{array}$ & Temporary & Not Applicable & $\begin{array}{c}\text { Waste Processing Tank } \\
\text { Buildings }\end{array}$ \\
\hline DISPOSAL: & None & None & $\begin{array}{l}\text { Shallow Land } \\
\text { Burial } \\
\text { Greater } \\
\text { Confinement } \\
\text { Disposal }\end{array}$ & $\begin{array}{l}\text { Hazardous/Mixed Waste } \\
\text { Disposal Facility (91) }\end{array}$ \\
\hline
\end{tabular}


The special case waste data and priority evaluations for each field office are shown in Tables 16 through 25 . The tables have been split into parts $A$ and $B$. Part $A$ of a Table gives details on the special case waste material and Part $B$ includes the data on the priority evaluation. The priority evaluations were performed assuming that all mixed waste is a regulatory problem for storage. A few comments on each of the field office tables follow.

Albuquerque - Some large diameter metal spheres containing plutonium need to be size reduced and packaged for shipment to the Waste Isolation Pilot Plant (WIPP). The walls are 2 or more inches thick and no facility is available at Los Alamos National Laboratory (LANL) for this work. LANL also has a Los Alamos Molten Plutonium Reactor Experiment reactor core, encased in concrete, that needs a final disposal facility identified. There is also some enriched uranium in various forms at LANL that should be sent to a recovery facility. A disposal method must be determined for some absorbed tritiated octane at Mound Laboratory.

Chicago - A disposal facility, somewhere in the DOE complex, for some nondefense transuranic (TRU) waste is needed. Performance assessments are needed to determine a disposal method and location for some other waste.

Idaho - A treatment facility for noncertifiable defense TRU waste is planned. A disposal facility for nondefense TRU waste and spent fuel is needed, and performance assessments are needed to determine a disposal method and location for some other waste.

Naval Reactors - Some characterization work is required to determine the extent of some of their problems. They are waiting for approval of a planned solidification facility for immobilization of some of their special case waste. 
TAHL IGA AL QUOUEROUE SPECIAL CASE UASTE (SCU) IMFORMATIOH

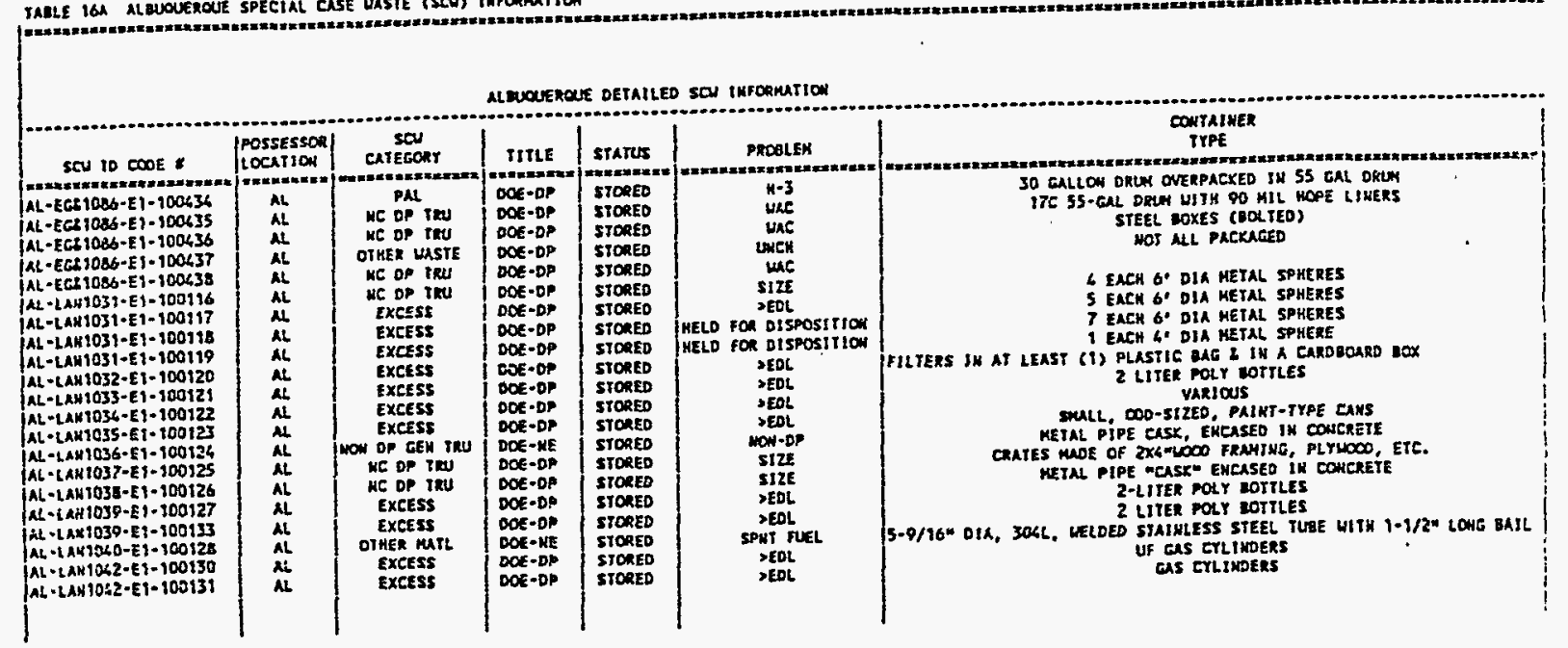

THEL 168 ALEUOUEROUE FIELD OFFICE SPEETAL CASE MASTE (SCW) PRIORITY EVALUATIOW

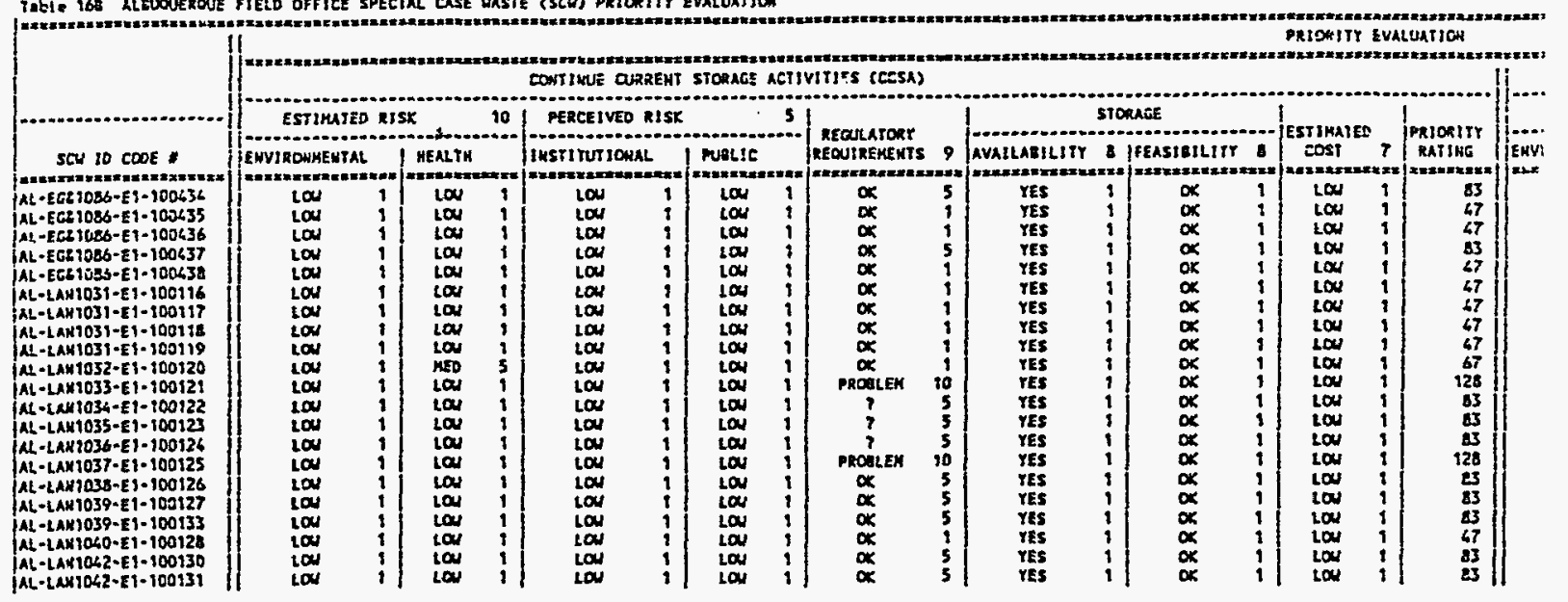




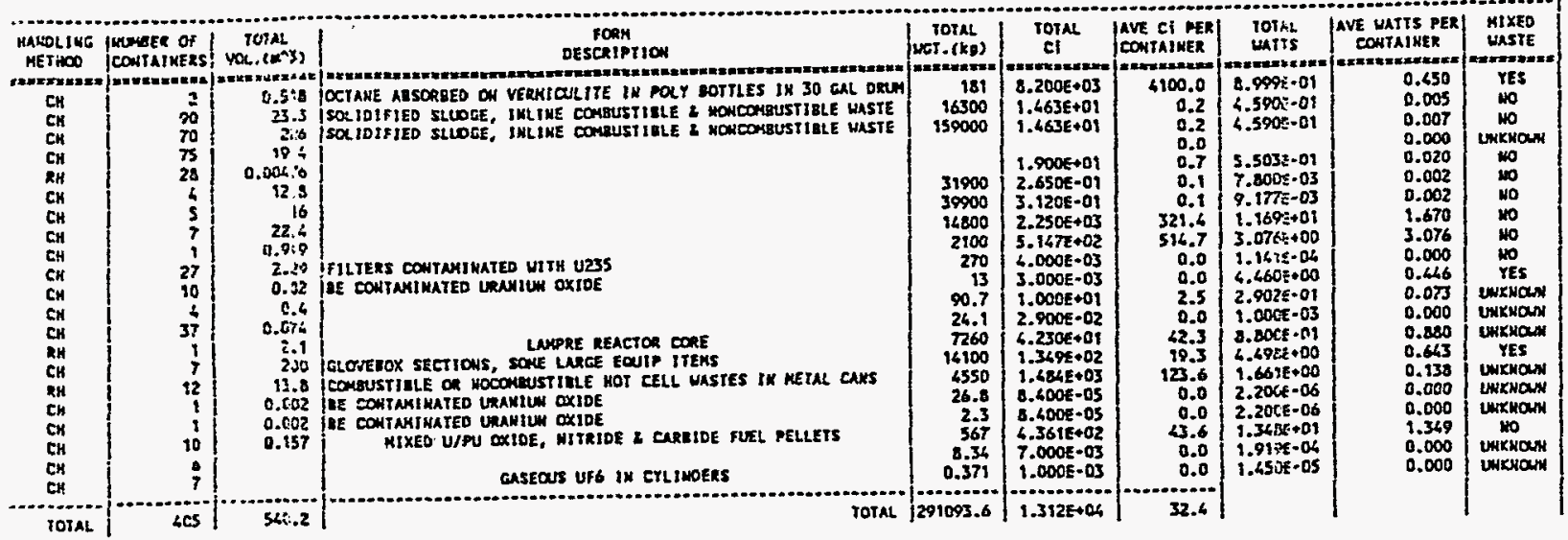

\begin{tabular}{|c|c|c|c|c|c|c|c|c|c|c|c|c|c|c|c|c|c|c|}
\hline \multicolumn{19}{|c|}{ 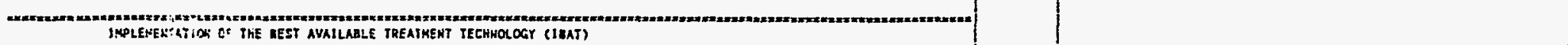 } \\
\hline SSTIN & 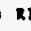 & & ic & \multicolumn{2}{|c|}{ EERCEIVED RISK } & & 51 & \multirow{2}{*}{\multicolumn{2}{|c|}{ 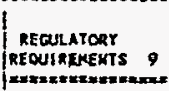 }} & \multicolumn{4}{|c|}{ TREATHEXT } & \multirow{2}{*}{\multicolumn{2}{|c|}{$\begin{array}{l}\text { ESTIMUTED } \\
\text { CosT }\end{array}$}} & \multirow[b]{2}{*}{ 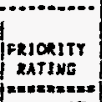 } & \multirow[b]{2}{*}{ 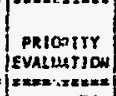 } & \multirow[b]{2}{*}{ POIEKTIAL RESOLUTIOH } \\
\hline POWreg & & 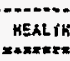 & & Trsitivitic & & malic & $=x=-1$ & & & AVAilatic & id & FERSIEIII & $a$ & & & & & \\
\hline tost & i & $t \alpha$ & 1 & tou & 1 & tov & 1 & ox & 3 & YES & 1 & $\quad \alpha$ & 1 & tou & 1 & 47 & -36 & PERFORK PA TO DETERAIKE DISPOSAL METHOO (TRITHLW) \\
\hline Lou & $i$ & MEs & 5 & LIM & 1 & LOS & 1 & ox & 1 & YES & 1 & TES & $i$ & ion & $i$ & 67 & 20 & REPACKACE TO KEET VIPP URE \\
\hline Lot & 1 & MED & 3 & Las & 1 & Lou & 1 & $\alpha$ & 1 & TES & 1 & YES & $i$ & Low & ; & 67 & 20 & REPACXAOE IO FII INTO IRUPACT II \\
\hline$\infty$ & 1 & MED & 5 & Las & 1 & LoN & 1 & $\alpha x$ & $i$ & $?$ & 5 & $\alpha$ & $i$ & MED & 3 & 127 & 44 & CMARACTERIZE WASTE TO DETERMINE DISPOSAL REQUIREHENTS \\
\hline 100 & 1 & MED & 3 & in & $i$ & $2 \infty$ & 1 & $\alpha x$ & $i$ & $i$ & 5 & $\alpha$ & $i$ & MED & 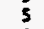 & 127 & 80 & REPAECAEE ANO SHIP TO UIPP (TEXCESS RTL) \\
\hline 104 & 1 & AEs & 5 & Low & 1 & LOW & 1 & $\alpha$ & $i$ & no & 10 & YES & 1 & K1GK & 10 & 202 & I3s & MEED KEW FACILIIY IO PROCESS VASTE \\
\hline La & 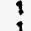 & MEC & 5 & Lou & 3 & 100 & $i$ & ơ & 1 & no & 10 & YES & t & MIGK & 10 & 202 & 155 & KEED KEU BACILITY TO PXOCESS WASTE \\
\hline 104 & 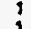 & MED & 5 & Low & 3 & Low & $?$ & $\alpha$ & ! & No & 10 & YES & 1 & HIEK & 10 & 202 & 155 & WEED MEW ERCILITY 10 PROCESS URSTE \\
\hline 100 & $!$ & KED & 5 & 104 & 1 & 100 & 9 & ox & 1 & no & 10 & VES & 1 & HIGK & 10 & 202 & 255 & NEED NEU FAEILITY TO MROCESS UASTE \\
\hline 10 & 1 & $\begin{array}{l}102 \\
\text { tout }\end{array}$ & 1 & 104 & 1 & 200 & 1 & ox & 1 & no & 10 & TES & $i$ & MEO & 5 & 167 & 80 & PACXUGE ANO SHIP TO TREATHENT FACILITT (93X U-235) \\
\hline 200 & i & KED & $\begin{array}{l}1 \\
5\end{array}$ & $\log$ & 1 & 104 & $i$ & 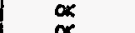 & ! & 40 & 10 & $\operatorname{trs}_{x \in S}$ & $\vdots$ & med & 5 & 147 & 19 & $\begin{array}{l}\text { PACXUGE AND SHIF TO IREATHENT FACILITY (93\% U-235) } \\
\text { PACEAGE AHD SHIP TO TREATMEMT FACILITY (U-233) }\end{array}$ \\
\hline $\begin{array}{lll}t a 2 \\
\operatorname{los}\end{array}$ & 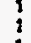 & $\begin{array}{l}\text { HED } \\
: E N\end{array}$ & 5 & $\operatorname{lom}$ & 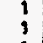 & low & 1 & $\stackrel{\alpha x}{\alpha}$ & 1 & No & $\begin{array}{l}16 \\
10\end{array}$ & $\begin{array}{l}\text { YES } \\
\text { YES }\end{array}$ & $\dot{q}$ & $\begin{array}{l}\text { MED } \\
\text { MED }\end{array}$ & 5 & $\begin{array}{l}167 \\
147\end{array}$ & 84 & 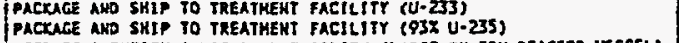 \\
\hline 104 & 1 & 20 & 1 & Las & $?$ & Lo4 & i & or & $i$ & yes & 9 & $\alpha$ & i & ton & i & 47 & .36 & WEED TO IDENTIFY A DISPOSAL FACILITY (LERGE RH TRU REACTOR VESSEL) \\
\hline LOW & $?$ & MED & 3 & Lo4 & 1 & La & 1 & PROBLEK & 10 & no & 10 & rES & i & MIEx & 10 & 203 & 855 & WEED REH FRCILITY TO PROCESS MUTERIAL \\
\hline 201 & $?$ & MES & 5 & 너 & 1 & tod & 1 & 7 & 5 & no & 10 & $?$ & 5 & HIEX & 10 & 270 & 137 & HOT CLEAR, ASSUME SIZE REDUCTIOH, SHIP TO YIPP \\
\hline tou & 1 & tov & 3 & 10 & 1 & tow & 1 & $\mathbf{\alpha}$ & $i$ & vo & to & res & $i$ & 104 & $i$ & 119 & 36 & PACEAGE WO SHIP IO TREATHEHT FACILITY (U-235) \\
\hline Lou & 1 & $\cos$ & 1 & La: & $i$ & Low & 1 & o* & i & no & 10 & rEs & 1 & 100 & 1 & 119 & 36 & \\
\hline $2 \infty$ & ! & 10 & $i$ & MED & 5 & MIEK & 10 & $\alpha$ & 1 & no & 10 & 7 & 5 & nick & 10 & 266.5 & 299.5 & WEED KEU DISPOSAL FACILLITY OK SEND TO RUCEA KTK \\
\hline $\operatorname{tow}$ & 1 & 10 & 1 & 10 & $!$ & 100 & 1 & $\mathbf{\alpha}$ & 1 & wo & 10 & YES & 1 & $\mathbf{t a}$ & 1 & 119 & 36 & $\begin{array}{l}\text { PACXACE ANO SHIP IO TREATHEKT FACILITY }(U-235) \\
\text { PACEAGE NOD SHIP TO }\end{array}$ \\
\hline $2 \alpha$ & & LaN & & $\operatorname{La}$ & & LOW & & & & No & 10 & YES & & tar & & 119 & & PACEAGE AND SHIP TO TREAIKEHT FACILITY (93X U-235) \\
\hline
\end{tabular}


TAELE 17A CHICACO SPEEIAL CASE WASTE (SCW) IHFORHATIOH

\begin{tabular}{|c|c|c|c|c|c|c|c|}
\hline \multicolumn{8}{|c|}{ CHICACO DETAILED SCH IYFORMTIOH } \\
\hline 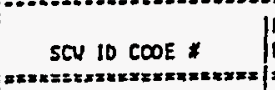 & $\begin{array}{l}\text { POSSES5OR } \\
\text { LOCATIOH } \\
|=E x=E x=x|\end{array}$ & 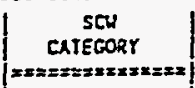 & $\begin{array}{c}\text { TITLE } \\
\mid x=\leq x= \pm \times=x=\end{array}$ & 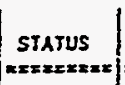 & $\begin{array}{c}\text { PROBLEH } \\
x=x==x=x x x=x=x=x=x=x 2\end{array}$ & $\mid$\begin{tabular}{c|} 
COHTA!NER \\
TYPE
\end{tabular} & 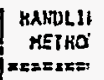 \\
\hline 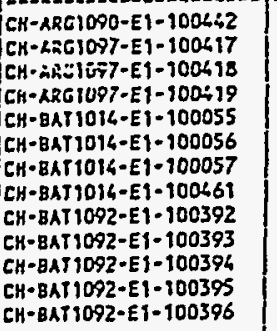 & \begin{tabular}{l|}
$\mathrm{CH}$ \\
$\mathrm{CH}$ \\
$\mathrm{CH}$ \\
$\mathrm{CH}$ \\
$\mathrm{CH}$ \\
$\mathrm{CH}$ \\
$\mathrm{CH}$ \\
$\mathrm{CH}$ \\
$\mathrm{CH}$ \\
$\mathrm{CH}$ \\
$\mathrm{CH}$ \\
$\mathrm{CH}$ \\
$\mathrm{CH}$
\end{tabular} & \begin{tabular}{|} 
HC OP TRU \\
SPAR \\
SPAR \\
PAL \\
WHOH DP GEN TRU \\
HOH OP GEN IRU \\
HOH DP GEN TRU \\
HOH DP CEN TRU \\
HOH OP GEH TRU \\
HOH DP GEH TRU \\
HOH DP GEH TRU \\
HOH DP EEN TRU \\
EXEESS
\end{tabular} & $\mid \begin{array}{l}D O E-D P \\
D O E-K E \\
D O E-K E \\
D O E-D P \\
D O E-K E \\
D O E-H E \\
D O E-K E \\
D O E-K E \\
D D E-K E \\
D O E-K E \\
D O E-K E \\
D O E-K E \\
D O E-K E\end{array}$ & \begin{tabular}{|l|} 
STORED \\
STORED \\
STORED \\
STORED \\
STORED \\
FUTURE \\
STORED \\
FUTURE \\
STORED \\
STORED \\
STORED \\
STORED \\
STORED
\end{tabular} & \begin{tabular}{|c|} 
TRMNSP \\
W235, 238, MUP, MFP -TRU \\
$5 R-90 \& C 5-137$ \\
KON-DP \\
KOH-DP \\
KOH-DP \\
NOH-DP \\
KOH-DP \\
KOH-DP \\
KOH-DP \\
KOH-DP \\
2EDL
\end{tabular} & 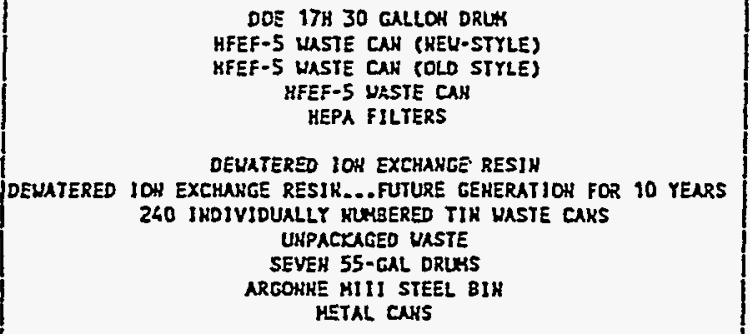 & $\begin{array}{l}\text { RH } \\
\text { CH } \\
\text { iH } \\
\text { CH } \\
\text { EH } \\
\text { EH } \\
\text { CHH } \\
\text { ZH } \\
\text { CH } \\
\text { iH } \\
\text { CH } \\
\text { CH } \\
\text { CH }\end{array}$ \\
\hline
\end{tabular}

TABle I7B CHICACO FIELO OFFICE SPECIAL CASE UASTE (SCN) PRIORITY EVALUATION

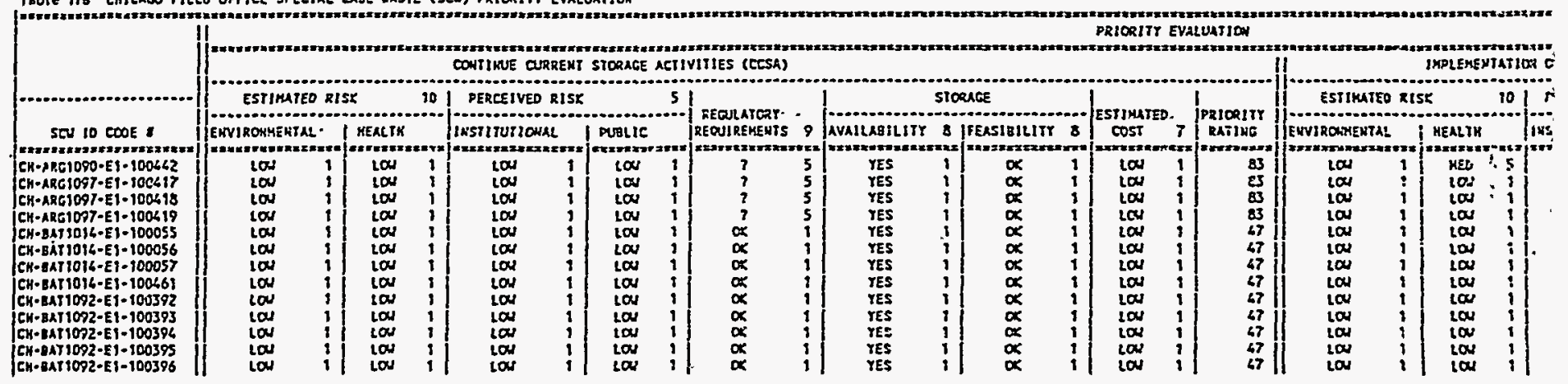




\begin{tabular}{|c|c|c|c|c|c|c|c|c|c|}
\hline$\sum_{n=2}^{i 6}$ & $\begin{array}{l}\text { HUABER OF } \\
\text { COHIAJHERS } \\
=2 \times 2 E x=2=2\end{array}$ & $\left\{\begin{array}{c}\operatorname{TOTAL} \\
\operatorname{Vot} \cdot\left(m^{n} 3\right)\end{array}\right.$ & 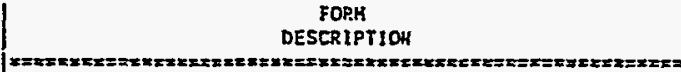 & 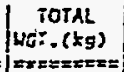 & 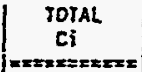 & 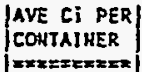 & 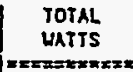 & 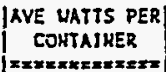 & 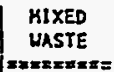 \\
\hline & $\begin{array}{r}45 \\
5 \\
359 \\
33 \\
1 \\
1 \\
25 \\
50 \\
260 \\
1 \\
7 \\
2 \\
2\end{array}$ & $\begin{array}{r}6.83 \\
0.65 \\
46.7 \\
4.29 \\
8 \\
15 \\
6.48 \\
13 \\
1.56 \\
3.56 \\
1.81 \\
6.94 \\
0.00379\end{array}$ & $\begin{array}{l}\text { SOL10 COHBUSTIBLE \& HONCONBUSTIBLE HATLS } \\
\text { TYPICALLY STAIHLESS STEEL PARTS \& HAROUARE } \\
\text { TYPICALLY STAIMLESS STEEL PARTS H HARDUARE } \\
\text { TYPJCALLY STAIHLESS STEEL PARTS I HARDUARE } \\
\text { HOT COMPACTED, METAL FRAHES \& AIR FILTERIHE MATERIAL }\end{array}$ & $\begin{array}{r}1400 \\
2500 \\
2900 \\
32500 \\
544 \\
4540 \\
730 \\
1690 \\
0.907\end{array}$ & $\begin{array}{l}7.840 E+01 \\
6.604 E+04 \\
7.338 E+06 \\
7.289 E+04 \\
6.397 E+01 \\
3.245 E+01 \\
1.165 E+01 \\
5.760 E+01\end{array}$ & \begin{tabular}{|r|}
13208.0 \\
20641.4 \\
2208.9 \\
64.0 \\
32.5 \\
0.5 \\
1.2 \\
0.0 \\
0.0 \\
5.4 \\
7.8 \\
0.4
\end{tabular} & $\begin{array}{l}3.600 E-02 \\
2.336 E+02 \\
3.841 E+04 \\
3.817 E+02 \\
6.270 E-01 \\
1.3800=01 \\
1.280 E-01 \\
5.200 E-01\end{array}$ & \begin{tabular}{|c|}
0.001 \\
46.721 \\
106.984 \\
11.563 \\
0.627 \\
0.138 \\
0.005 \\
0.010 \\
0.000 \\
0.009 \\
0.186 \\
0.287 \\
0.014
\end{tabular} & $\begin{array}{l}\text { NO } \\
\text { YES } \\
\text { YES } \\
\text { YES } \\
\text { KO } \\
\text { UHKHOWN } \\
\text { NO } \\
\text { HO } \\
\text { NO } \\
\text { HO } \\
\text { YO } \\
\text { HO } \\
\text { HO }\end{array}$ \\
\hline & $\pi 1$ & 116.8 & & 149944.90 & $7.478 E+06$ & 9698.7 & & & \\
\hline
\end{tabular}

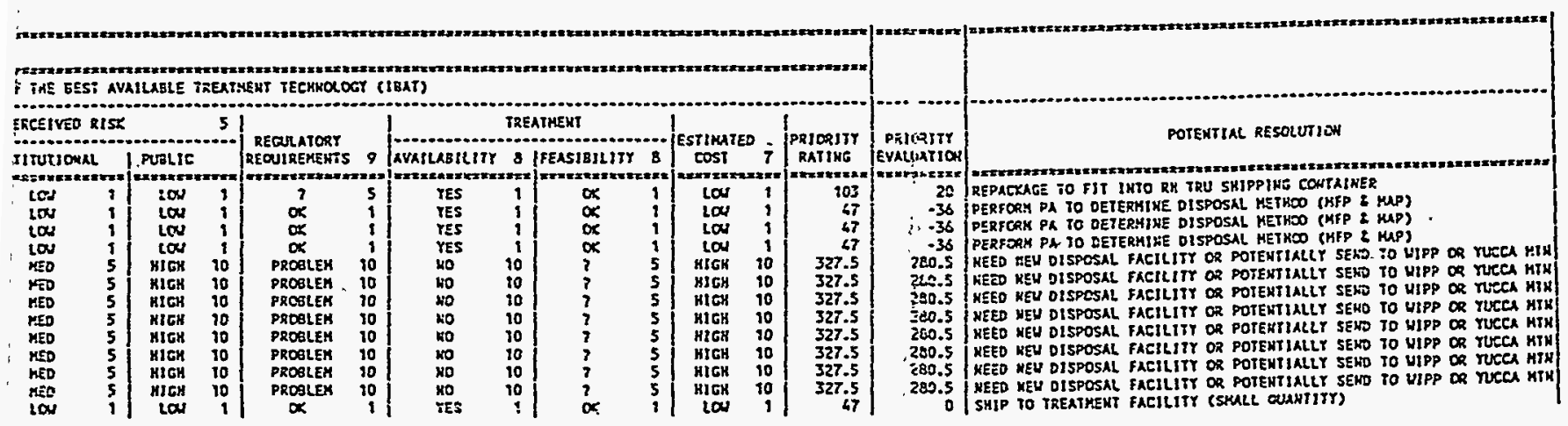




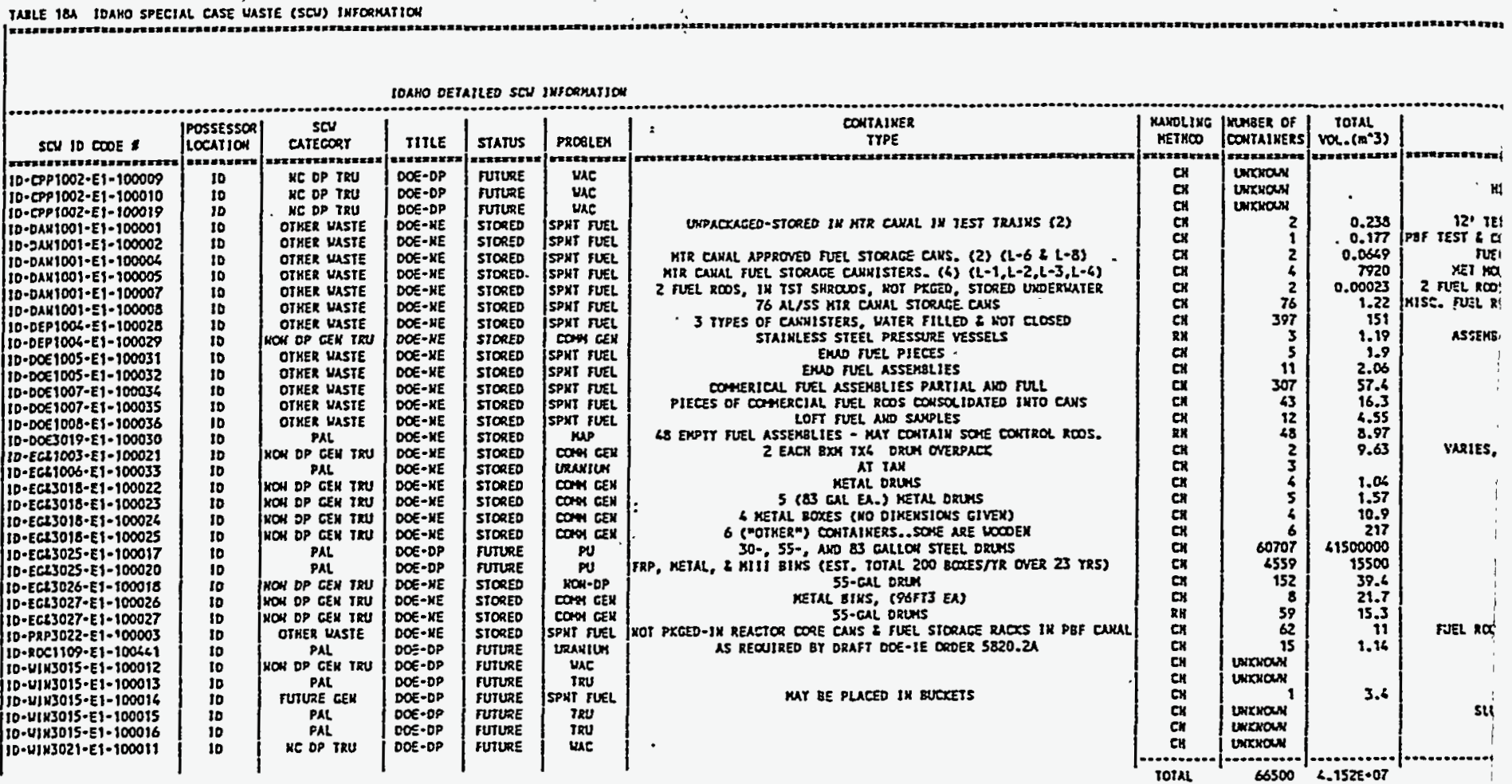

THble 188 JDAHO FIELD OFFITE SPECIAL CASE UASTE (SCU) PRIORITY EVALUATION

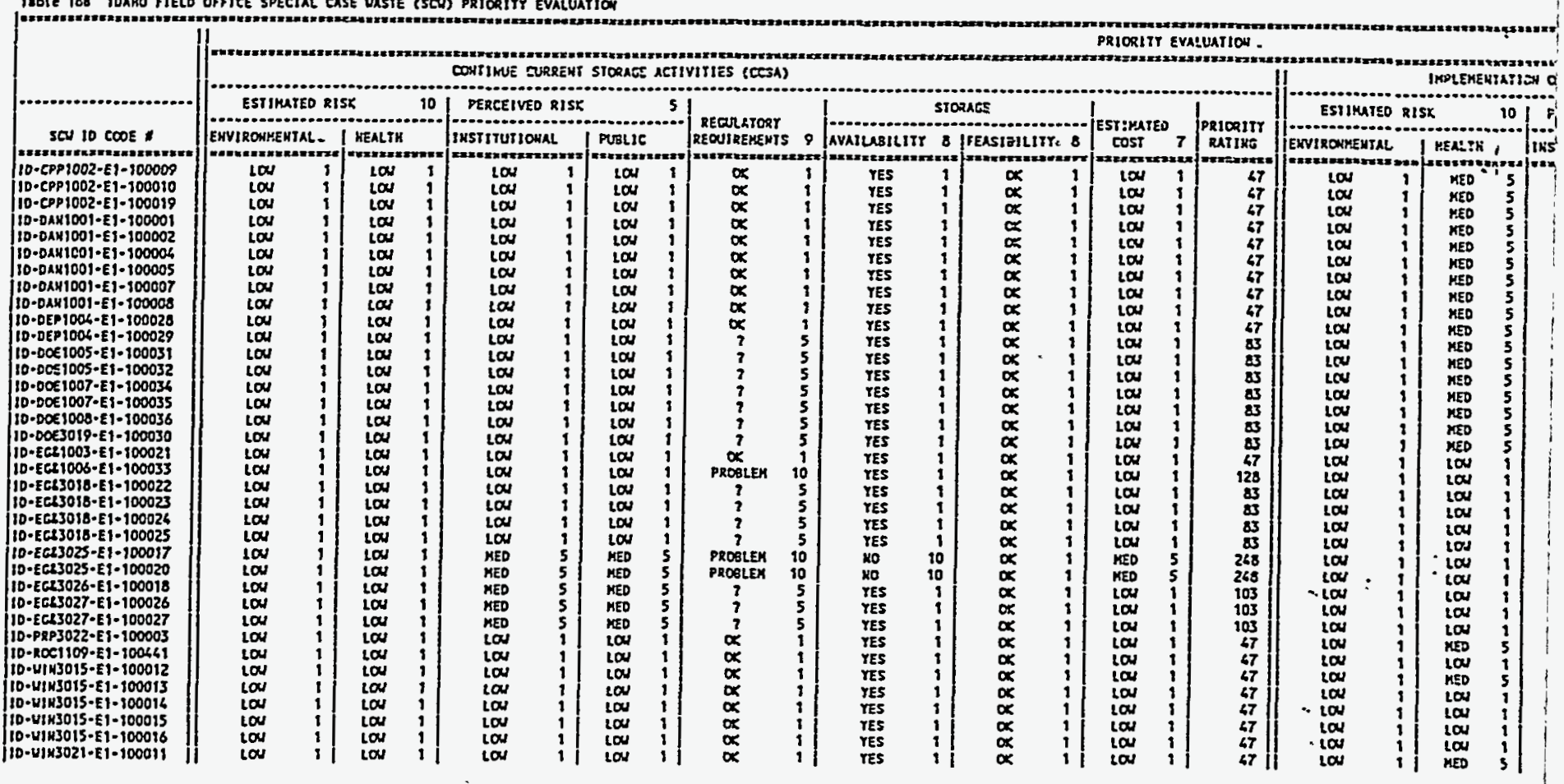




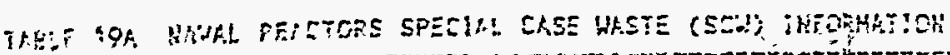

(1)

\begin{tabular}{|c|c|c|c|c|c|c|c|}
\hline \multicolumn{8}{|c|}{ HAVAL PEACTORS DETAILED SCU INFORHATIOH } \\
\hline 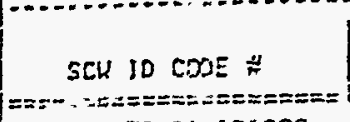 & 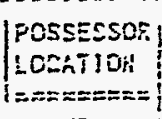 & 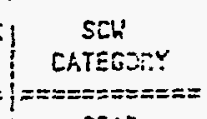 & I:YYE & 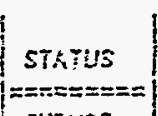 & $\begin{array}{l}\text { FROELEH } \\
========\end{array}$ & 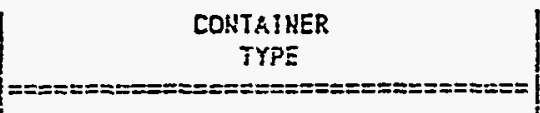 & $\begin{array}{l}\text { HANDLIHG } \\
\text { METHOO } \\
======:=-1\end{array}$ \\
\hline & $\begin{array}{ll}\operatorname{lin} \\
\mathrm{kin}\end{array}$ & SPsin & $\begin{array}{l}0,-2 E \\
0,-20\end{array}$ & 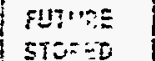 & TPAP & 55 EAL DRUYSS \& SS BOXES & $\begin{array}{l:}\mathrm{CH}_{\mathrm{H}} \\
\mathrm{RH}\end{array}$ \\
\hline 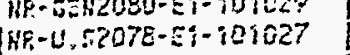 & $\begin{array}{l}\text { Kri } \\
H R\end{array}$ & SPh: & $\cos x-5$ & FUTijo & MAO & & $\mathrm{Ch}$ \\
\hline HQR-hE:208i-E:-:01030 & $:: f$ & GOTHEF WRSTE & {$[1 \div-20$} & STOEED & $\mathrm{MECH}$ & 316 STAIHLESS STEEL TAKKS & CH \\
\hline WF-MES2082-Eq-70T03i & in? & |OTYER WHSTE & DOE-S? & FLTUDE & UNCH & & RH! \\
\hline WK- $4 E=2082-51-101032$ & HP. & IOTKER WH.STE & $00=-.20$ & DTHER & UHCK & TO BE DETERMINED & $R H$ \\
\hline$H R-L E S 20 E ?-E:-90: 033$ & WR & WC DP TRU & $D O E-5 p$ & STORES & WAE & UHPACKAGED & RH \\
\hline$K P \cdot-U E 52082-E T-101034$ & KR. & SPAR & DoE-D? & FUTL?E & & 55 TOH SCRAP EASK IUSERTS & $\begin{array}{ll}\mathrm{CH} \\
\mathrm{CH}\end{array}$ \\
\hline$H F_{i}-i+2 E Z 0 E 2-E i-101035$ & HRR & SPAR & $D 0=-2 x$ & $s+0 \sigma_{0}=0$ & & & \\
\hline & & & & & & & \\
\hline
\end{tabular}

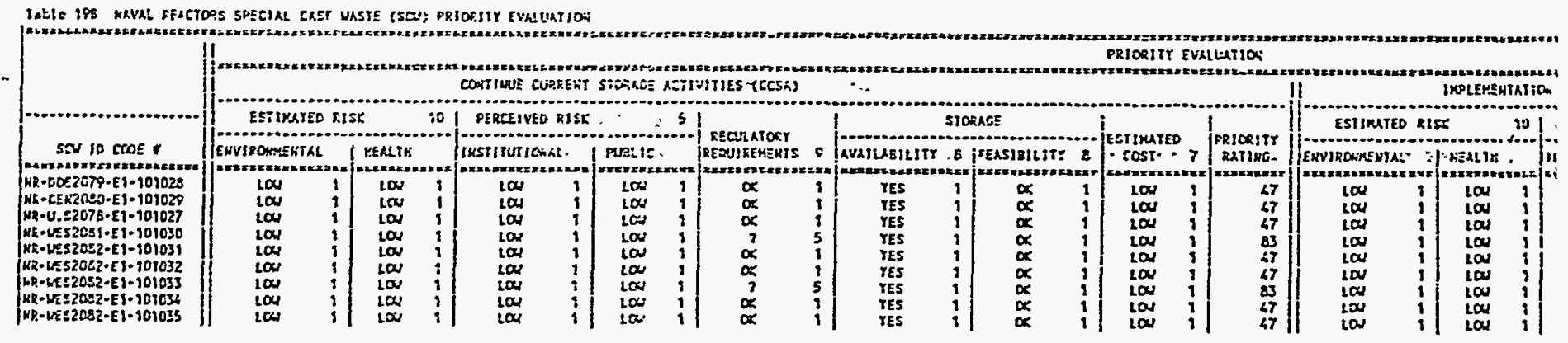




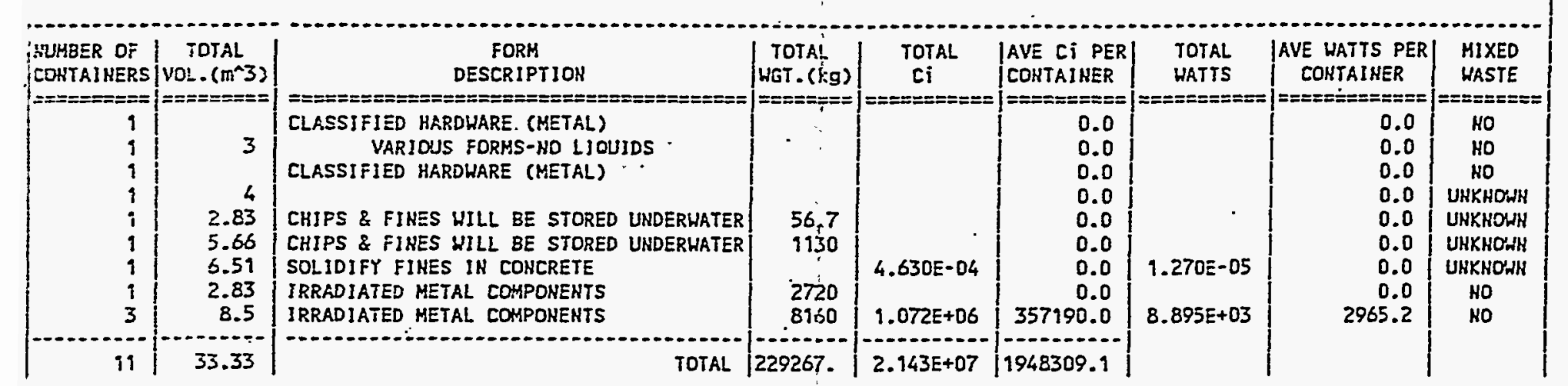

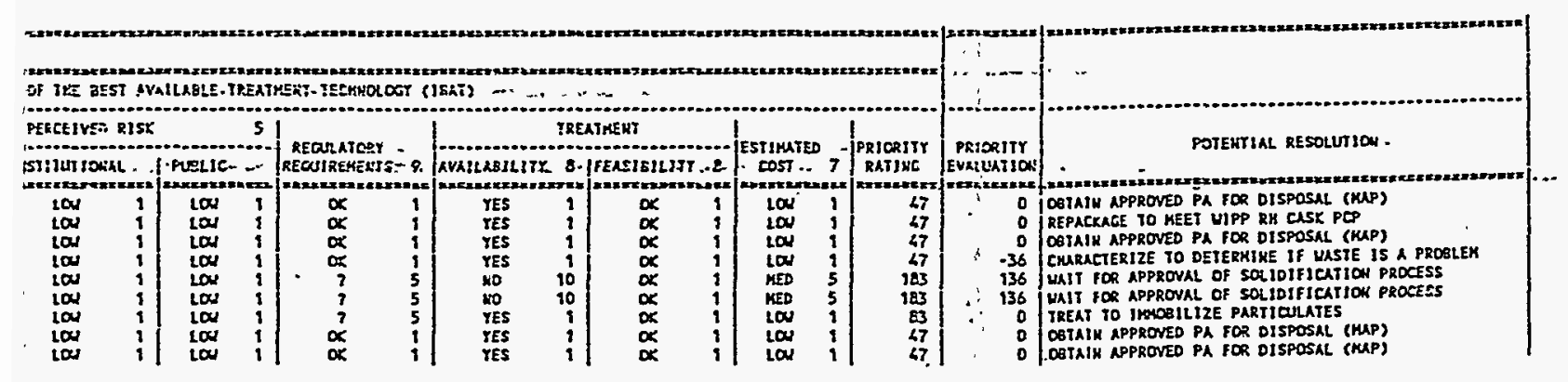


TABLE 20A HEVADA SPECIAL CASE HASTE (SCH) INFORHATION

\begin{tabular}{|c|c|c|c|c|c|c|c|c|c|}
\hline , & & & NEVADA DET & SAILED SCH & INFORHATIOH & & & & \\
\hline SEU ID COOE $\#$ & $\begin{array}{l}\text { POSSESSOR } \\
\text { LOCATIOH } \\
\text { I=== }\end{array}$ & $\mid \begin{array}{c}\text { SCW } \\
\text { CATEGORY. }\end{array}$ & TITLE & $=\mid \begin{array}{l}\text { STATUS } \\
========1\end{array}$ & PROBLEH & $\mid \begin{array}{c}\text { COHTAIMER } \\
\text { TYPE } \\
========\end{array}$ & $\mid \begin{array}{c}\text { HANDLIHG } \\
\text { METHOO }\end{array}$ & $\begin{array}{l}\text { |NUMBER OF } \\
\text { CONTAINERS| } \\
|=======0|\end{array}$ & $\left\{\begin{array}{c}\text { TOTAL } \\
\text { VOL. }\left(m^{\wedge} 3\right)\end{array}\right.$ \\
\hline 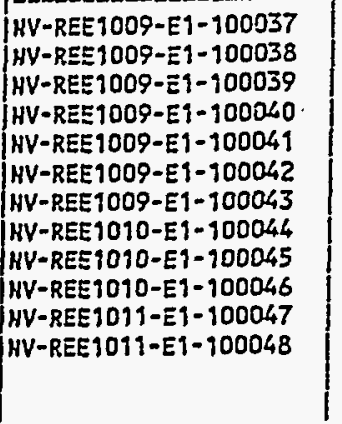 & $\begin{array}{l}\text { HV } \\
H V \\
W V \\
H V \\
H V \\
H V \\
W V \\
H V \\
H V \\
H V \\
H V \\
H V\end{array}$ & 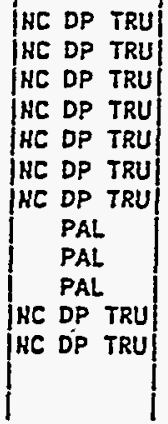 & \begin{tabular}{|l}
$D O E-D P$ \\
$D O E-D P$ \\
$D O E-D P$ \\
$D O E-D P$ \\
$D O E-D P$ \\
$D O E-D P$ \\
$D O E-D P$ \\
$D O E-D P$ \\
$D O E-D P$ \\
$D O E-D P$ \\
$D O E-D P$ \\
$D O E-D P$
\end{tabular} & \begin{tabular}{|} 
TDISPOSED \\
STTORED \\
OISPOSED \\
DISPOSED \\
STORED \\
TOISPOSED \\
STORED \\
STORED \\
STORED \\
STORED \\
STORED \\
STORED
\end{tabular} & \begin{tabular}{|c|} 
CLASFD \\
CLASFD \\
CLASFD \\
CLASFD \\
CLASFD \\
CLASFD \\
CLASFD \\
URAKIUH \\
URAKIUK \\
UURAHIUH DAUGHTERS \\
HAC \\
TRANSP
\end{tabular} & $\mid \begin{array}{c}55-G A L \text { DRUMS- } \\
55-G A L \text { DRUH } \\
55-G A L \text { DRUMS } \\
55-G A L \text { DRUH } \\
55-G A L \text { DRLA } \\
\text { 55-GAL DRUM } \\
\text { 6-H DRUMS } \\
55-G A L \text { DRUH } \\
\text { WOOOEN BOX } \\
\text { WOODEH BOX } \\
\text { 55-GAL DRUM } \\
\text { METAL BOXES }\end{array}$ & $\begin{array}{l}\mathrm{CH} \\
\mathrm{CH} \\
\mathrm{CH} \\
\mathrm{CH} \\
\mathrm{CH} \\
\mathrm{CH} \\
\mathrm{CH} \\
\mathrm{CH} \\
\mathrm{CH} \\
\mathrm{CH} \\
\mathrm{CH} \\
\mathrm{CH} \\
\text { TOTAL }\end{array}$ & \begin{tabular}{|r|}
152 \\
210 \\
74 \\
102 \\
38 \\
32 \\
47 \\
8 \\
217 \\
51 \\
207 \\
58 \\
1196
\end{tabular} & $\begin{array}{r}39.4 \\
54.4 \\
19.2 \\
26.4 \\
9.84 \\
8.29 \\
2.4 \\
2.07 \\
633 \\
9240 \\
53.6 \\
273 \\
10361.6\end{array}$ \\
\hline
\end{tabular}

TESLE 208 MEYROA TEST SITE SPEE!AL CASE UASTE (SEY).PRIORITT EVALUATIOH.

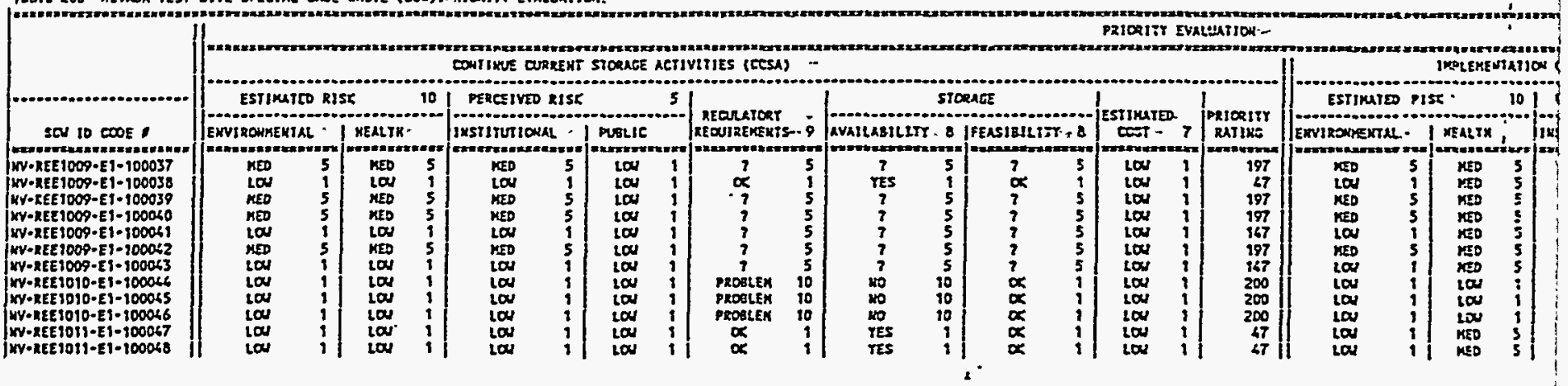




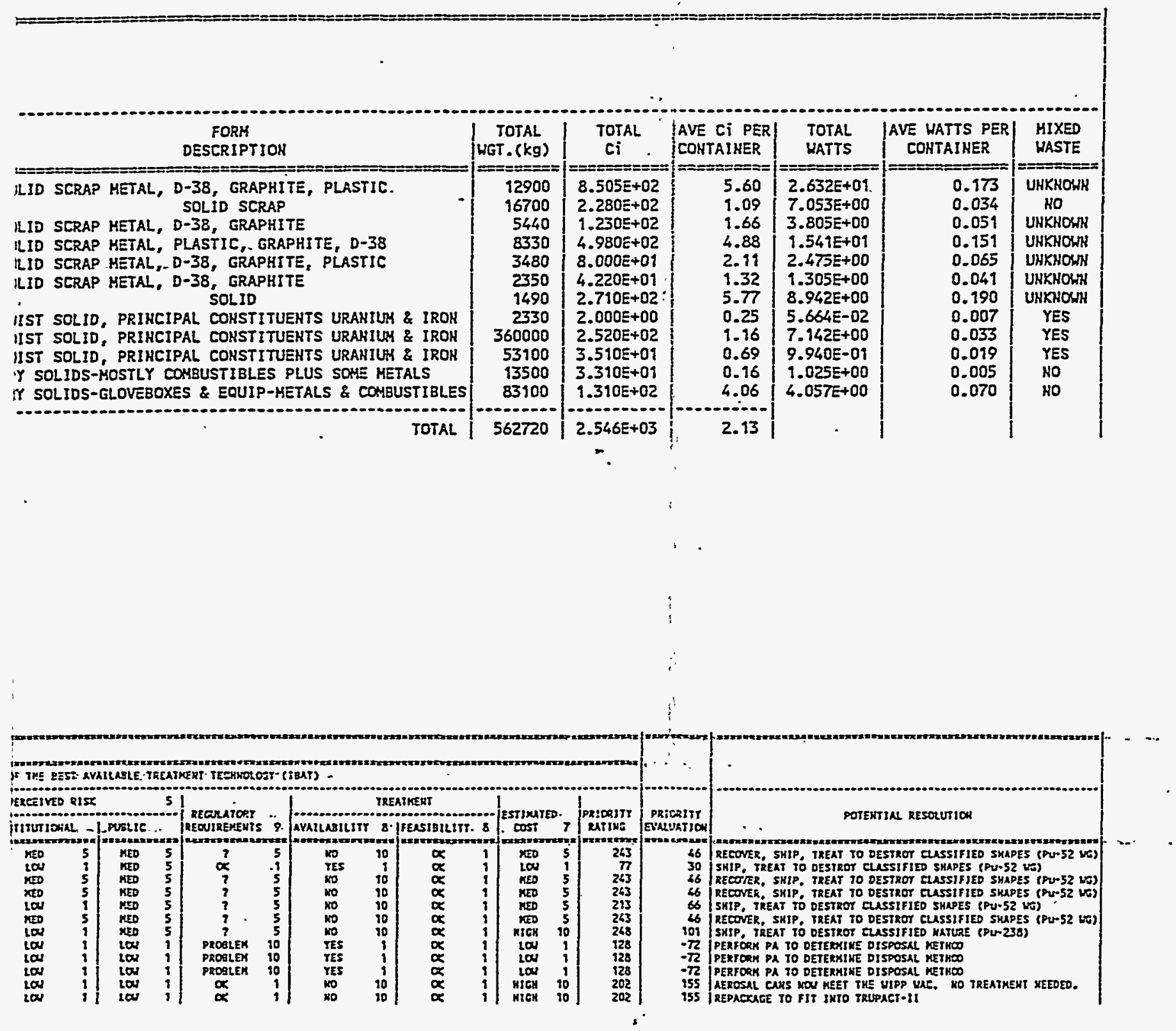




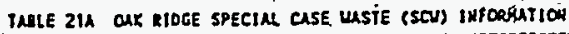

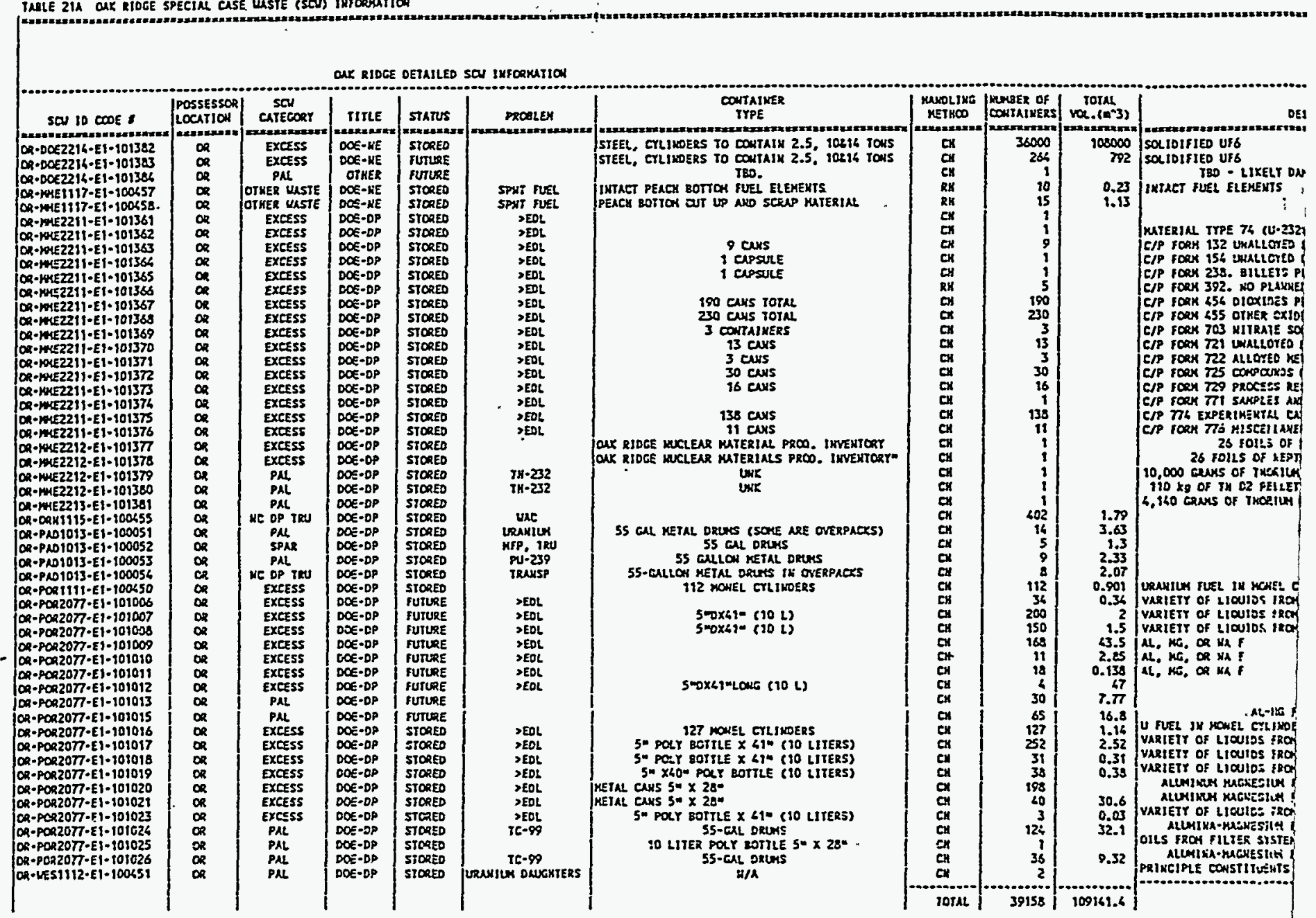




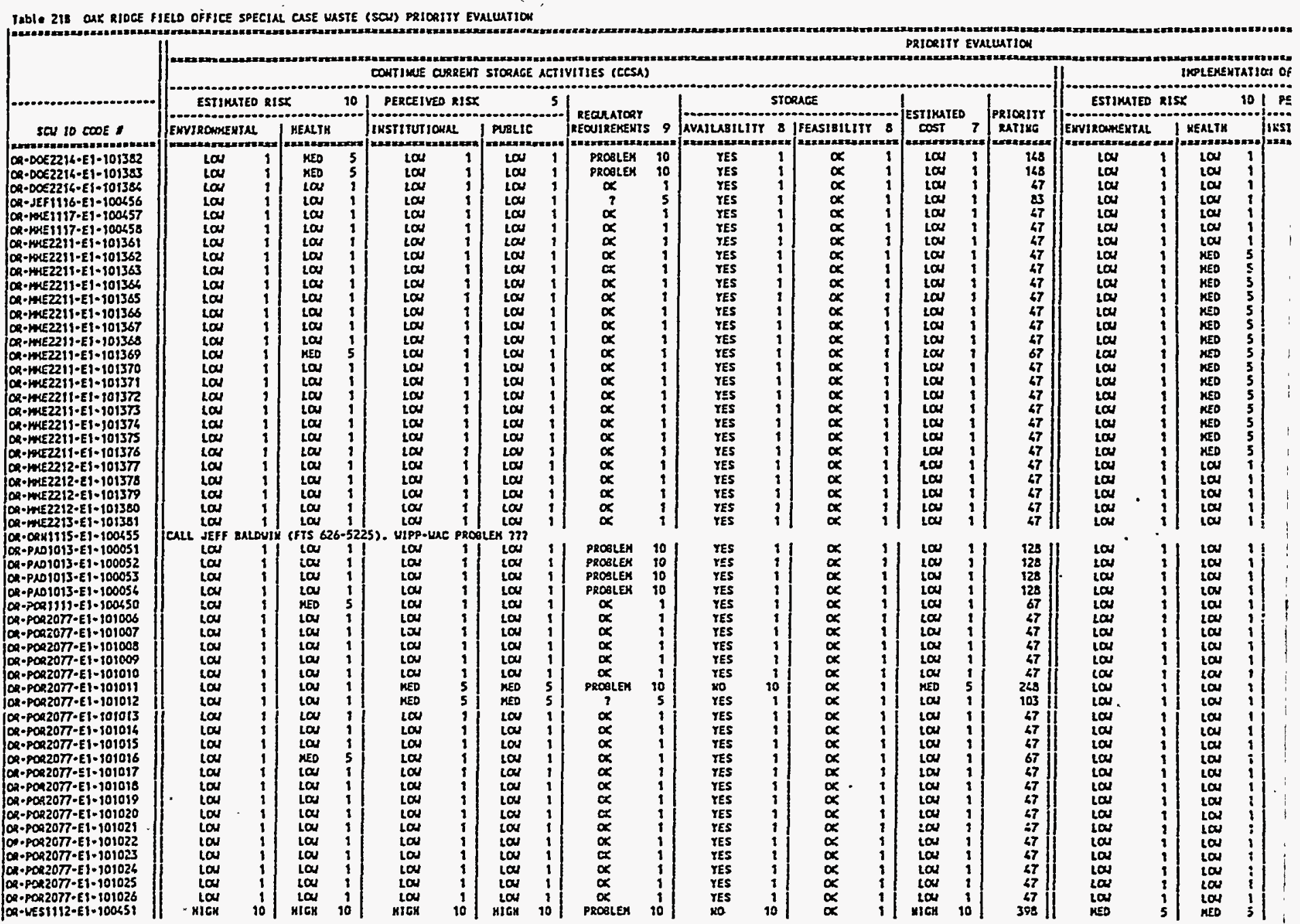




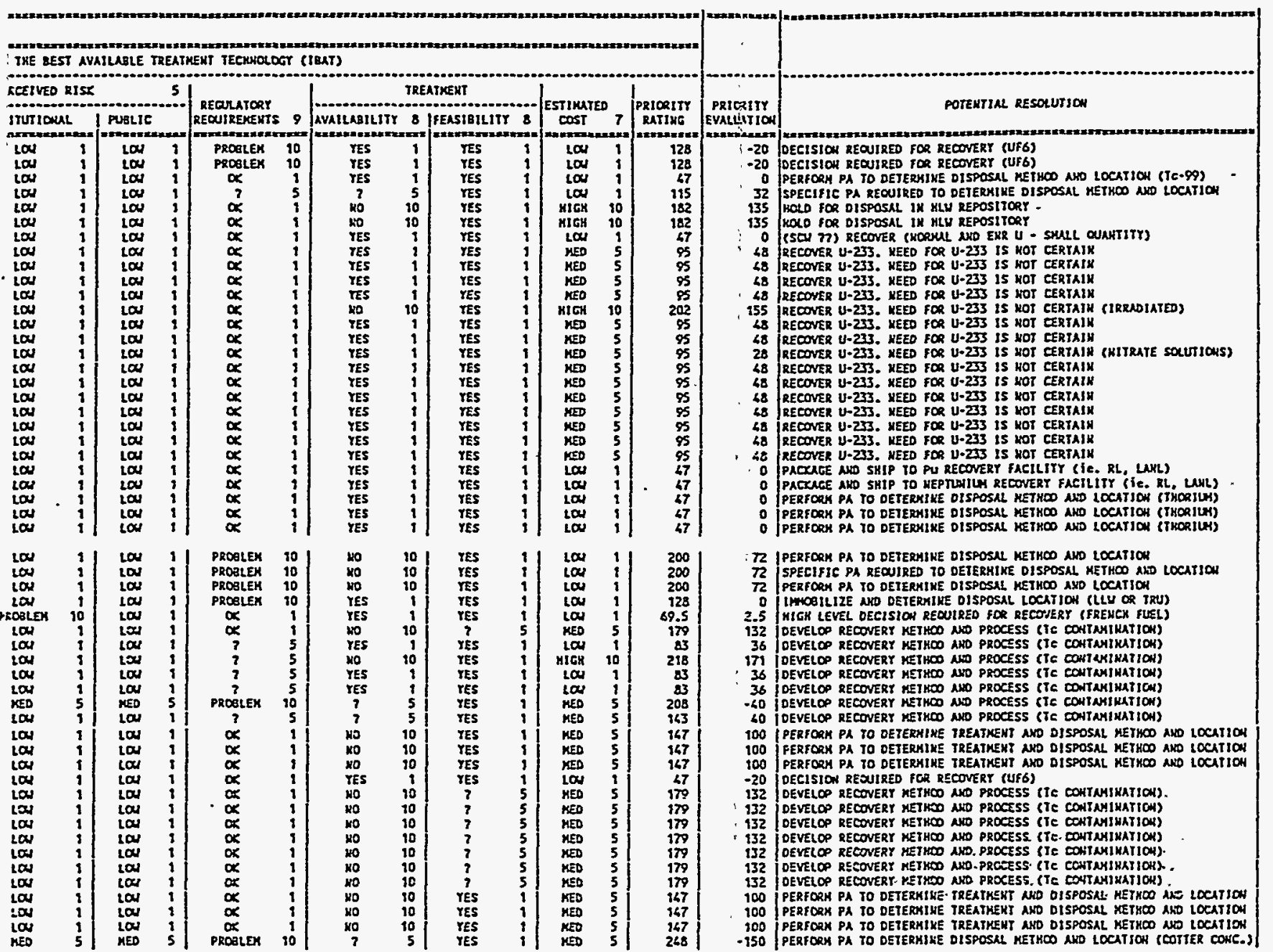


TABLE 22A RICKLAKD SPECIAL CASE WASTE (SCU) IHFORHATIOH

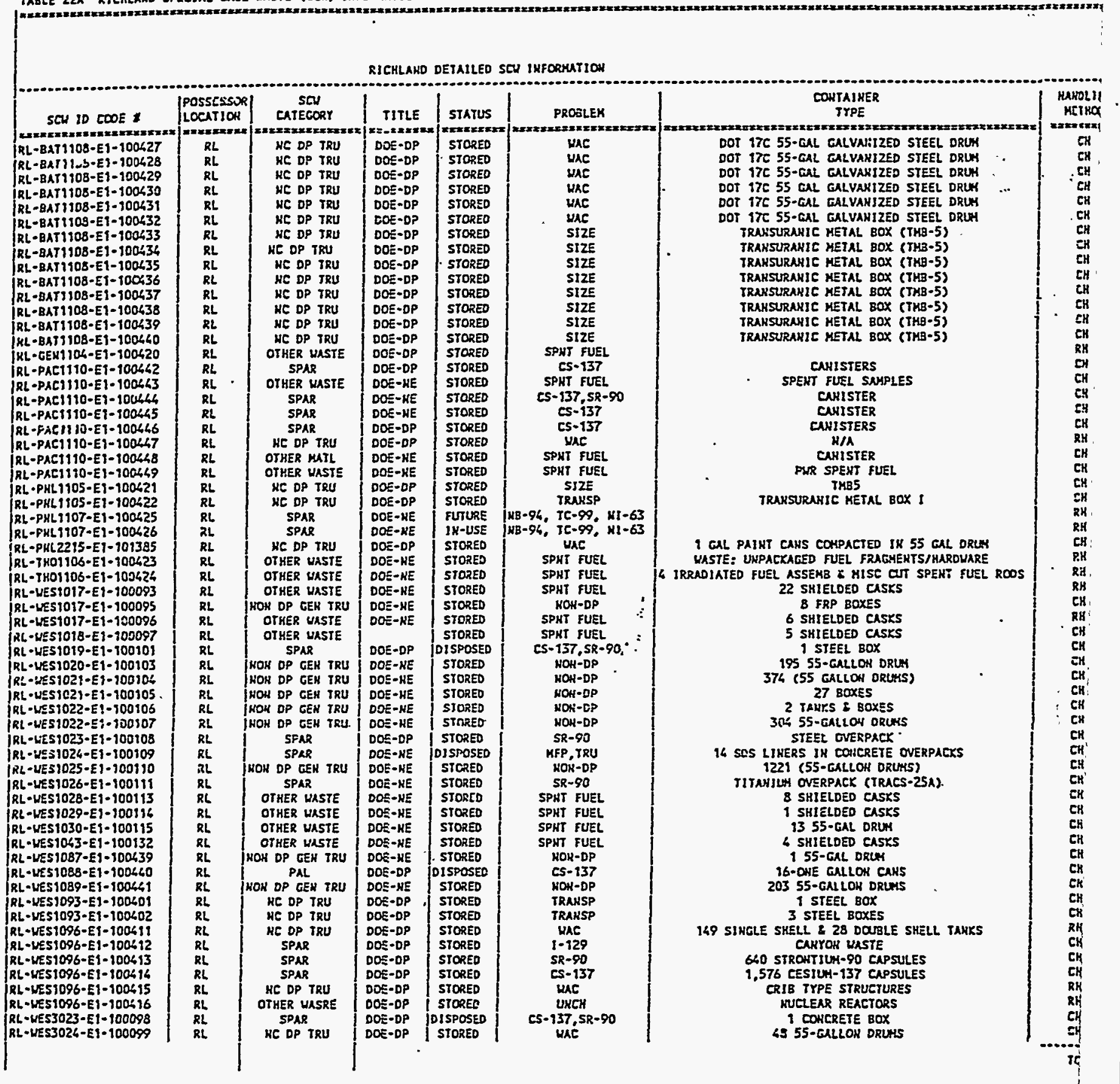


TAbl. 228 RICNLANO FIELO OFFICE SPECIAL CASE LUSTE (SCW) PRIORITY EVALUATION

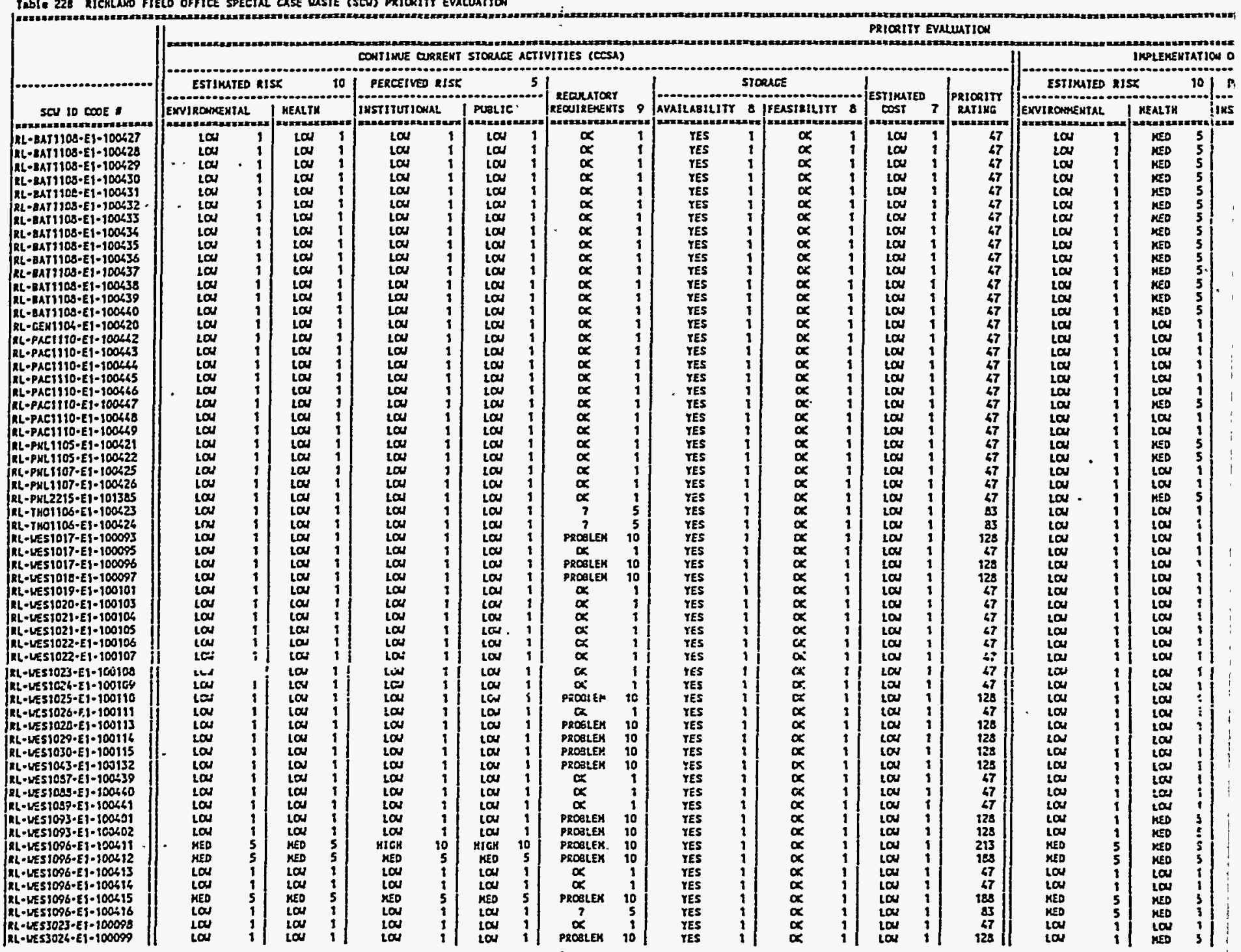




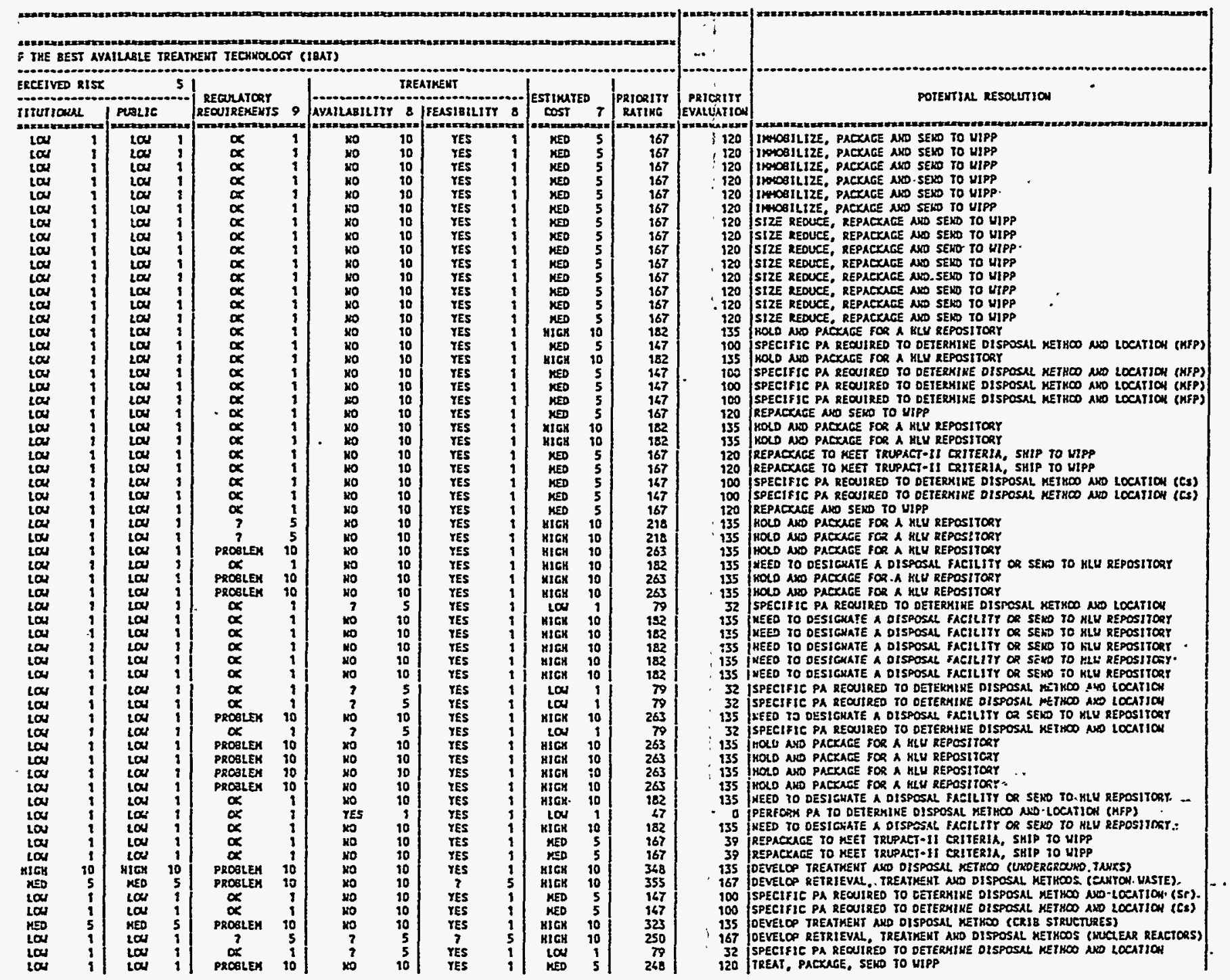


Table 23A ROCKY FLATS SPECIAL CASE HASTE (SCW) IHFORMATION,

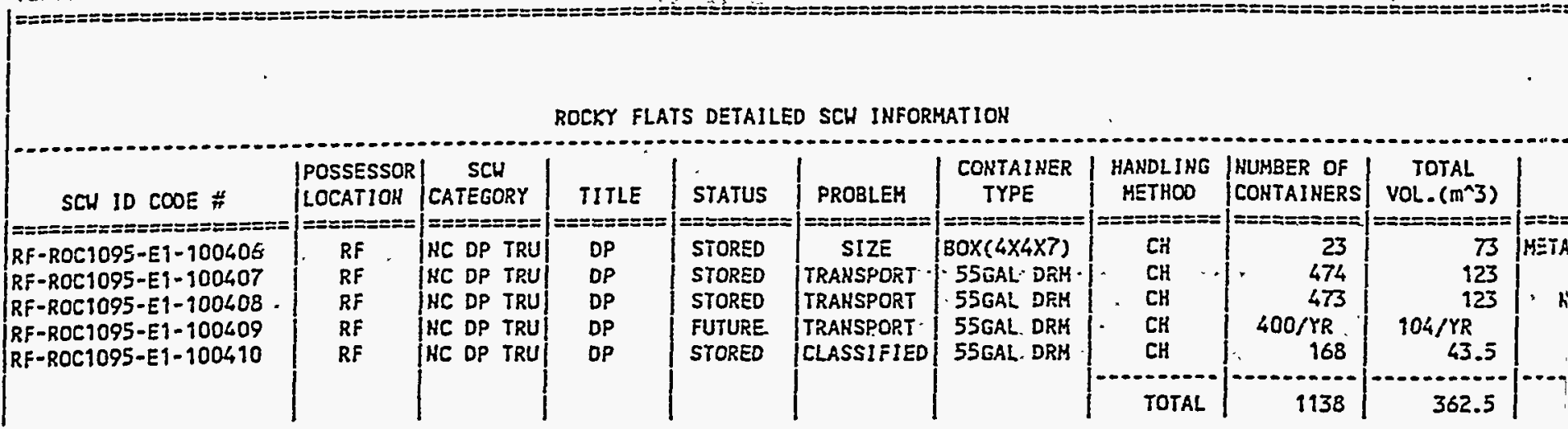

ROCKY FLATS DETAILED SCH INFORMATION

\begin{tabular}{|c|c|c|c|c|c|c|c|c|c|c|c|c|c|}
\hline \multicolumn{13}{|c|}{ 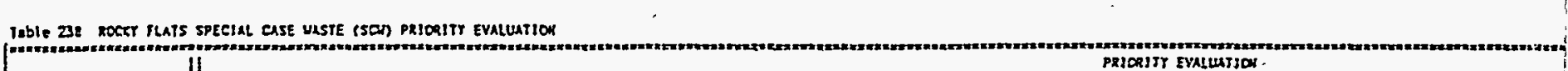 } & \\
\hline \multirow{3}{*}{ sed 10500,} & & & & & & & & & & & \multicolumn{3}{|c|}{ IMPLEVEXTAT:TY } \\
\hline & ESTIKUTED , & $15 x$ & If PERCEIVEO RISE & & 5 & 1 & stoe. & & & ipromert & ESTHKA:ES 2: & $: 5 \pi$ & 3014 \\
\hline & ENVIROOWENTAR & KEx<T: & JxSIITNTICNA1 & I mustse. & KEOUIREMENTS .9 & avalusustrt & 81 & FENS:BILITT-8 & $=052 \quad 7$ & RATSKE & EVVIROMUEWTR & HERLTX & ins \\
\hline 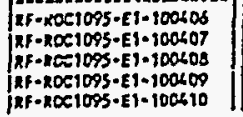 & $\begin{array}{l}\text { Lo } \\
\text { Lo } \\
\text { tow } \\
\text { tod }\end{array}$ & $\begin{array}{ll}\log & 1 \\
\log & 1 \\
\log & 1 \\
\log & 1 \\
\cos & 1\end{array}$ & 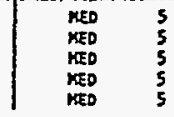 & $\begin{array}{ll}\text { KIEK } & 10 \\
\text { HEK } & 10 \\
\text { XIER } & 10 \\
\text { HER } & 10 \\
\text { KICX } & 10\end{array}$ & $\begin{array}{cc}\text { PROLLEK } & 10 \\
\text { PROSLEK } & 10 \\
\text { PROBLEK } & 10 \\
\text { PROSLEK } & 10 \\
\text { X } & 1\end{array}$ & $\begin{array}{l}\text { YES } \\
\text { YES } \\
\text { YES } \\
\text { YES } \\
\text { YES }\end{array}$ & $\begin{array}{l}1 \\
1 \\
1 \\
1 \\
1\end{array}$ & $\begin{array}{ll}\text { SPACE } & 10 \\
\text { SPACE } & 10 \\
\text { SPACE } & 10 \\
\text { SPAEE } & 10 \\
\text { SPACE } & 10\end{array}$ & $\begin{array}{ll}\operatorname{lo} & 1 \\
20 & 1 \\
2 \infty & 1 \\
2 \infty & 1 \\
2 \infty & 1\end{array} \mid$ & $\begin{array}{l}222.5 \\
222.3 \\
222.5 \\
222.5 \\
151.5\end{array}$ & $\begin{array}{l}\operatorname{lo} \\
\operatorname{lo} \\
\operatorname{la} \\
\log \\
\log \end{array}$ & 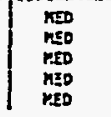 & $\begin{array}{l}5 \\
5 \\
5 \\
5 \\
5\end{array}$ \\
\hline
\end{tabular}


TABLE 2LA SAH FRAHCISCO SPECIAL CASE UASTE (SCW) IKFORMATIOH

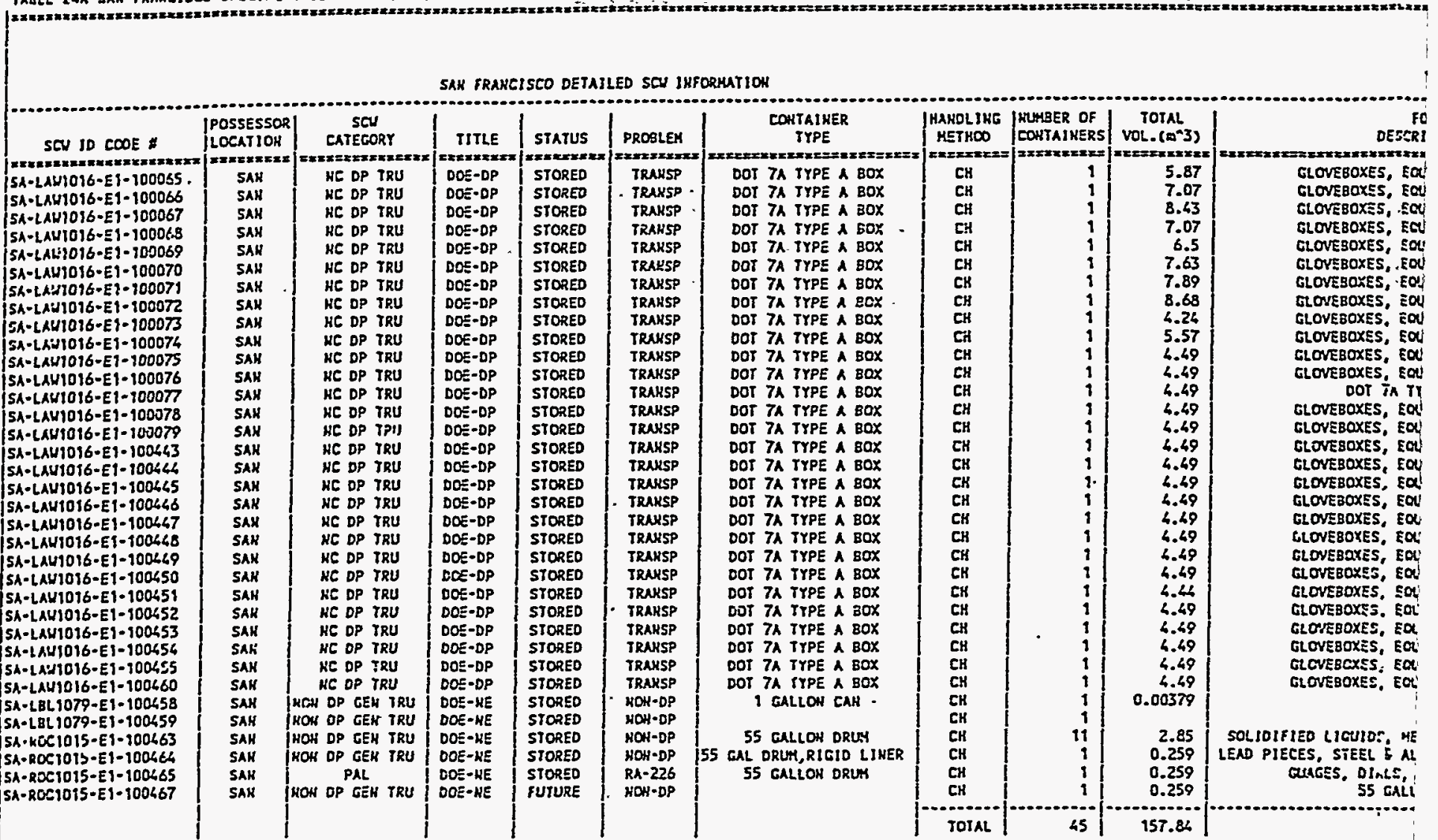

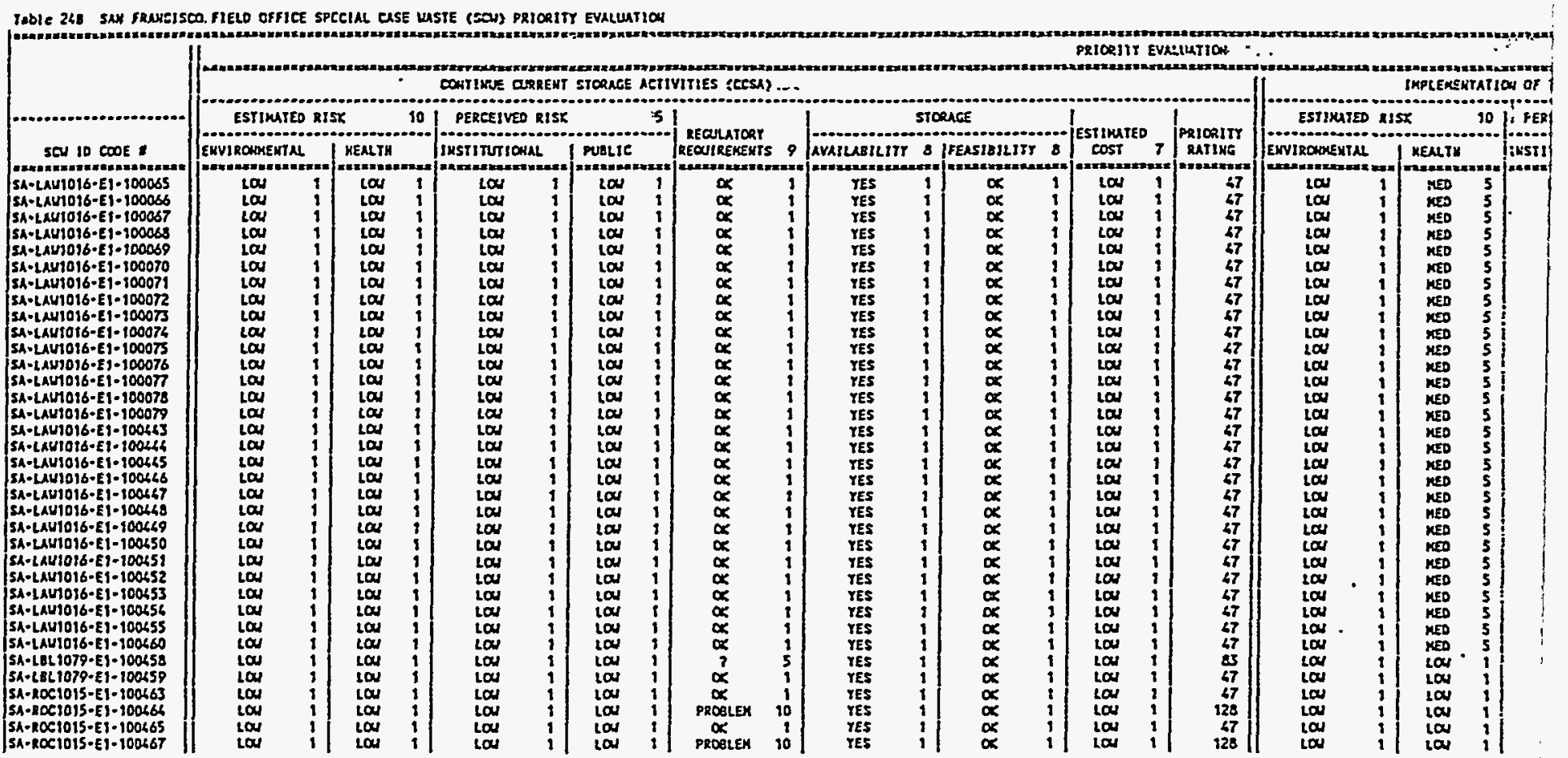


TABLE 25A SAVAHHAH RIVER SPECIAL CASE HASTE (SCH) IHFCRHATIOH

SAVAHKAH RIVER DETALLED SCH IMFORHATIOH

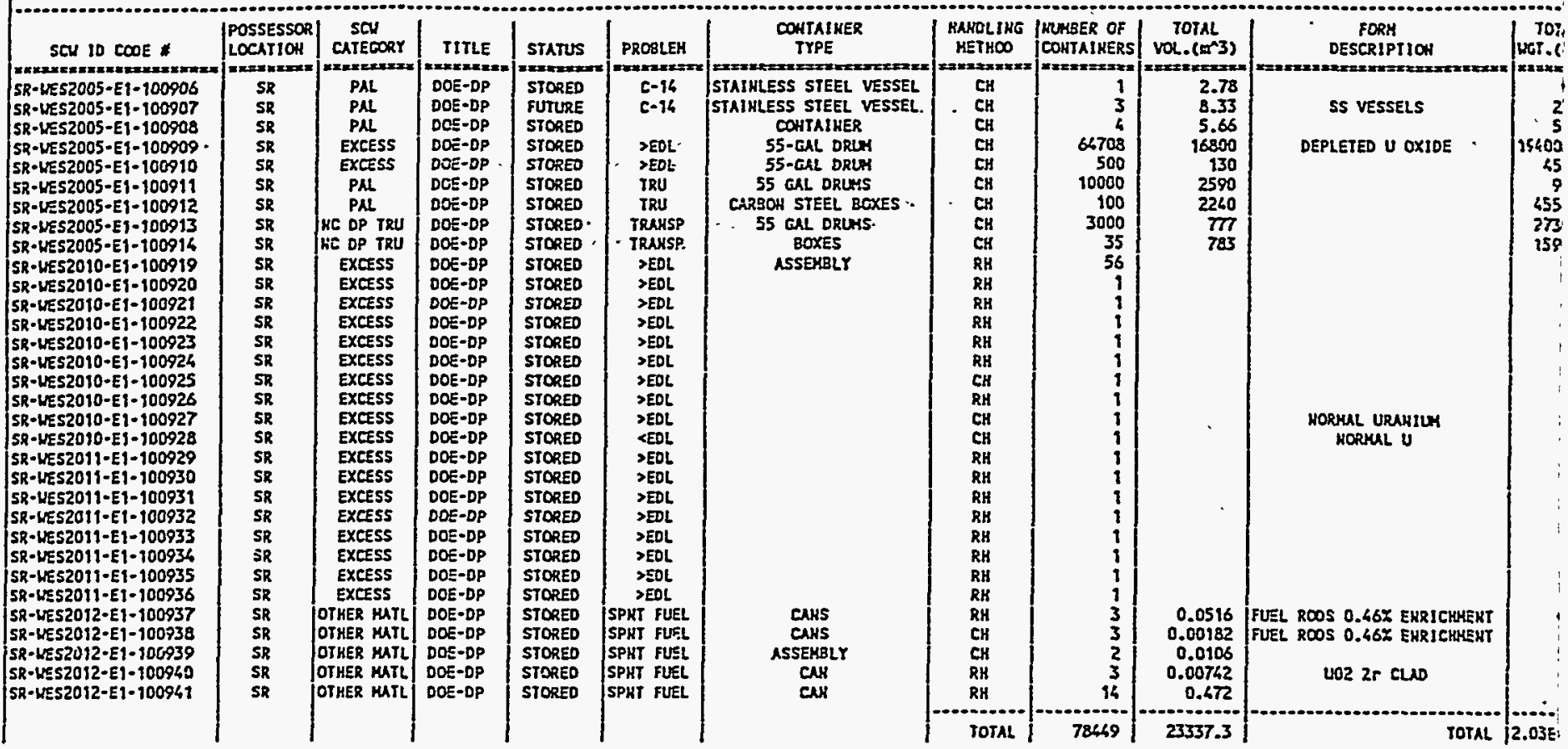

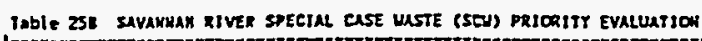

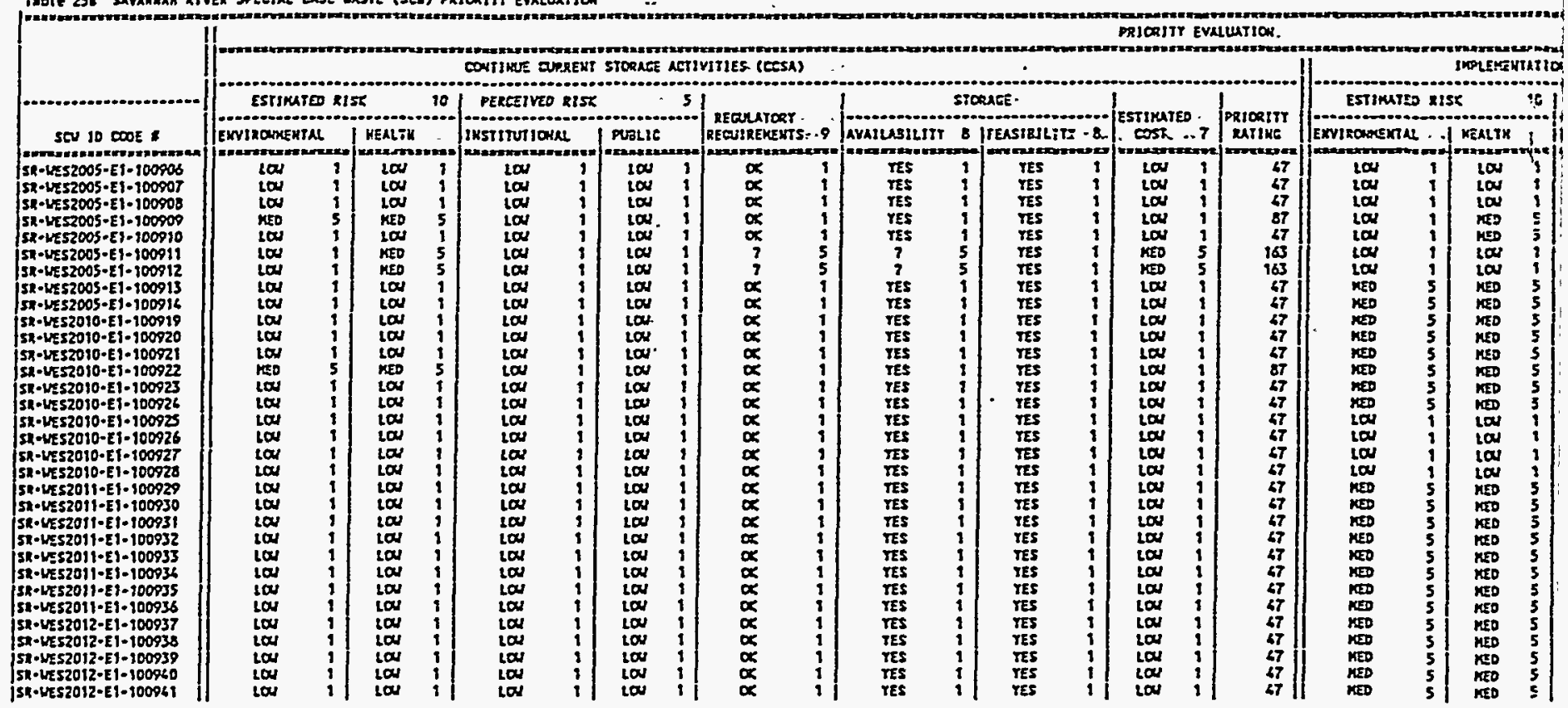


Nevada Test Site - Some TRU waste containing classified items must be recovered from shallow land burial and shipped to a facility to destroy the classified nature of the waste. Some performance assessments must be done to determine an acceptable disposal method and location for waste items containing radium.

Oak Ridge - There is some U-233 waiting for recovery, but the current need for it is uncertain. Some technetium contaminated uranium is also waiting recovery, but a recovery method must be developed. Performance assessments must be done to determine a disposal method and 7ocation for some of the waste.

Richland - Some of their waste is waiting for completion of the planned waste receiving and packaging facility so it may be treated and sent to WIPP. Performance assessments are needed to determine a disposal method and location for some waste items. A disposal facility must be designated for some nondefense TRU waste items. Methods for retrieval, treatment and disposal of some of their large, difficult to manage items (e.g., underground tanks, crib structures, nuclear reactors, and canyon waste) must be developed, funded, and approved.

Rocky Flats - A treatment facility is needed to destroy the classified nature of some items before they are discarded. Because Rocky Flats has a waste storage capacity problem, they are packaging waste to minimize storage volume. However, this packaging may not meet the TRUPACT-II Payload Compliance Plan; therefore, some treatment and repackaging of waste will be required when the waste can be moved.

San Francisco - Lawrence Livermore National Laboratory needs a facility to size reduce some defense TRU waste so the waste can be sent to WIPP. A disposal facility for some nondefense TRU waste, at Lawrence Berkeley Laboratory and Rockwell International, Canoga Park, must be determined.

Savannah River - The priority evaluations for several wastes indicate CCSA even though the material is greater than the economic discard limit. This is 
because there are no treatment or recovery facilities for this material, and recovery facilities will be very costly. Some of this material is waiting for the Multi-Purpose Processing Facility to open. Some defense TRU waste is waiting for the Transuranic Waste Facility to open for repackaging. Some excess material containing depleted and normal uranium was waiting for the Feed Materials Production Center to resume operation, but it now appears to have no recovery process available. A few waste items need a performance assessment to determine disposal method and location. 


\section{OVERALL PRIORITY EVALUATION RANKING}

Performing the priority evaluations for the special case waste items and examining the potential resolutions reveals that 22 resolutions are common throughout the DOE complex. The 22 resolutions identified in the priority evaluations were assigned a letter designation and are 1isted in Table 26. These letter designations were used in the spreadsheet where the special case waste items were sorted by priority evaluation ranking. The highest and lowest ranked items are shown in Tables 27 and 28 . Since these are only partial results all of the potential resolutions do not appear in the Tables. Table 27 lists the highest ranked special case waste items for all field offices for continuing storage. Table 28 lists the highest ranked special case waste items for a11 field offices for implementing treatment. 
Table 26. SCW potential resolution key

A Certify at Stored Waste Examination Pilot Plant and send to WIPP

B Characterize to determine if waste is a problem

C Decision required for recovery

D Designate a disposal facility or send to HLW repository

E Destroy classified nature, send to. WIPP

$F \quad$ Develop recovery method and process

$G$ Develop retrieval, treatment, and disposal methods

$H \quad$ Evaluate recovery value of uranium

I High level decision required for recovery

$\mathrm{J}$ Identify a disposal facility (Non DP TRU)

$K \quad$ Immobitize, package, and dispose (LLW) (RH)

L Immobilize, package, and send to WIPP

$M \quad$ Immobilize and determine disposal location (LLW or TRU)

$\mathrm{N} \quad$ Package and ship to recovery facility

0 Perform PA to determine disposal method and location (PAL)

$P \quad$ Recover, ship, treat to destroy classified nature

$R \quad$ Repackage and send to WIPP

$S \quad$ Ship, treat to destroy classified nature

$T \quad$ Size reduce, repackage and send to WIPP

$\mathrm{U}$ Specific PA required to determine disposal method and location

$\checkmark$ Treat, package, and send to WIPP

W Recover isotopes 
TABLE 27. HIGHEST RAMKED SCH ITEHS FOR COHTIHUED STORAGE (ALL FIELD OFFICES)

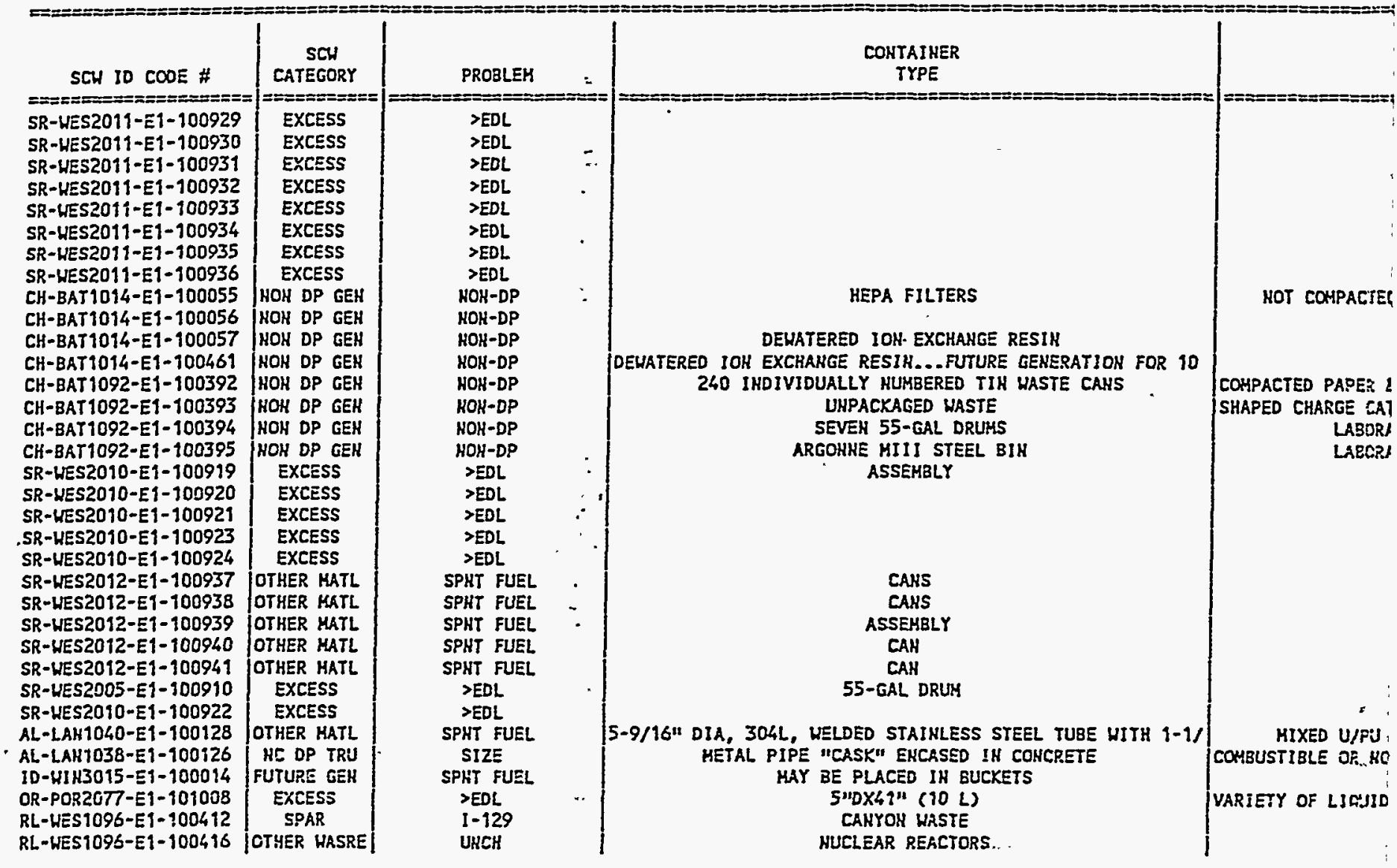




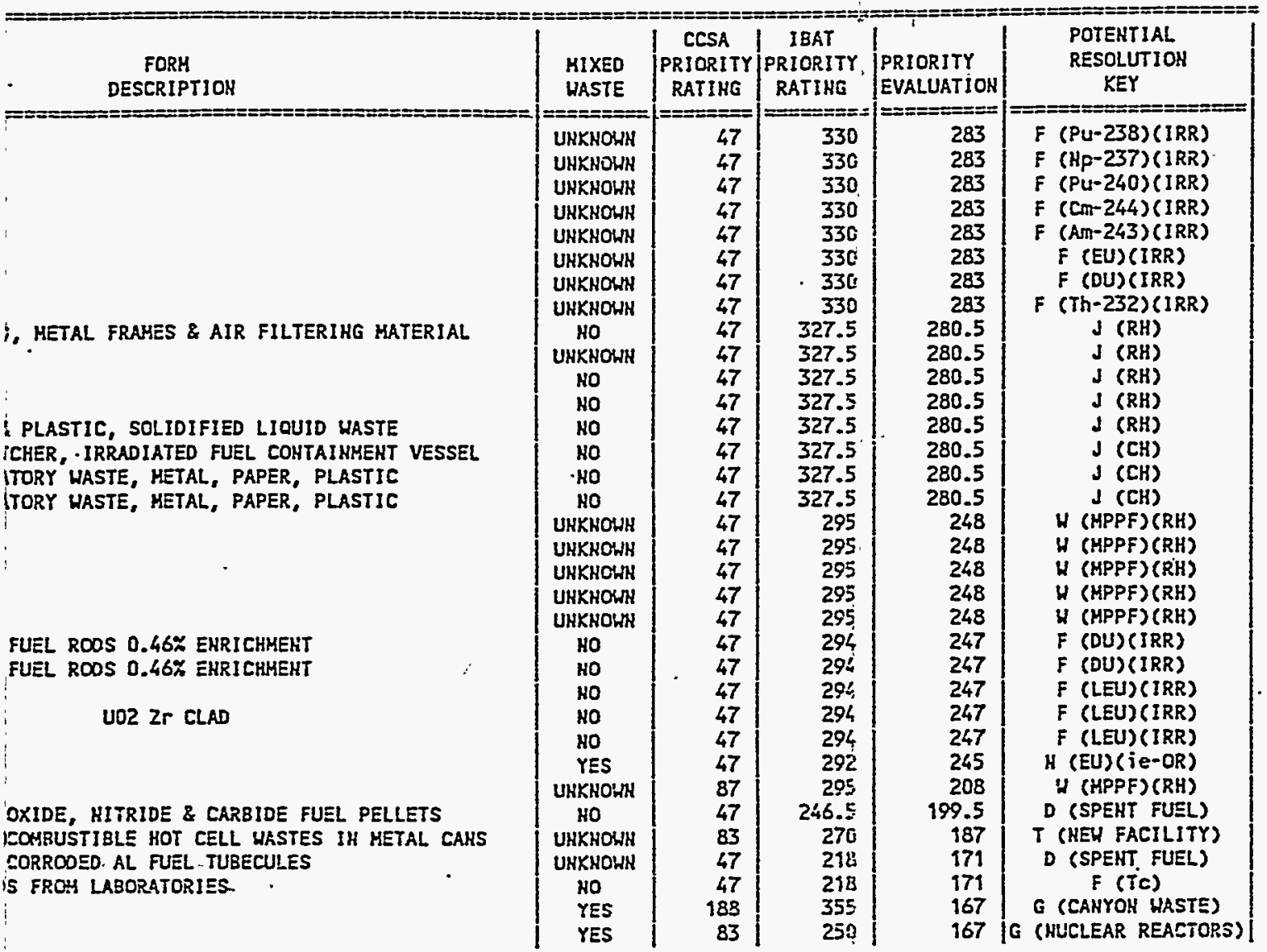


TABLE 28. HIGHEST RAHKED SCH ITEHS FOR IHPLEMENTIHG TREATHENT (ALL FIELD OFFICES)

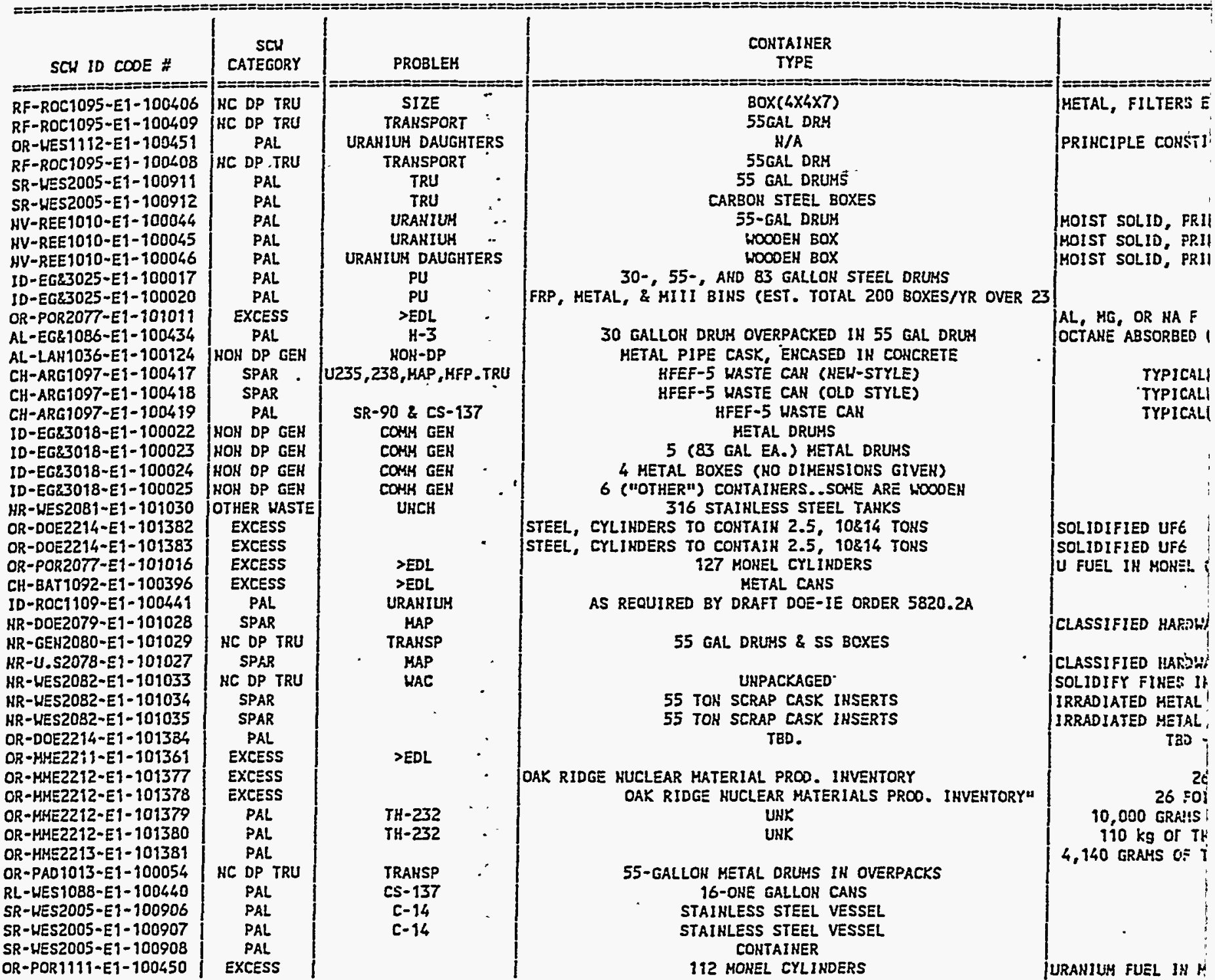




\section{RESULTS}

The highest ranked items for continued storage, shown in Table 27, are items that require development of a suitable recovery method for excess material or treatment methods for waste. Some of these recovery or treatment methods will require new facilities that are very costly, others are waiting for planned facilities, that will also be very costly to complete. The high cost of building new treatment facilities increased the priority rating for treatment of the waste. This drives the priority evaluation toward continuing storage.

Other items that also ranked high for continued storage are some nondefense TRU waste that need a disposal facility identified. This ranking depends on whether the nondefense TRU waste can be disposed at some existing facility or whether a new disposal facility will have to be built. The priority evaluation on these items assumed that a new disposal facility would have to be built. If a decision can be made to dispose of these items at some existing disposal facility, such as WIPP, then the priority evaluation would change dramatically.

The highest ranked items for implementing treatment, shown in Table 28, are items that need to be repackaged, certified, or characterized at an existing facility, and items that need to have a PA completed to determine the disposal method and location. It is possible that the results of the certification, characterization or PA could indicate that an expensive treatment is required to dispose of the waste. A new priority evaluation done at that time would give a different ranking.

There are also some excess materials that can be recovered at existing facilities. Some of these materials may have to be packaged and sent to other facilities for recovery where the capabilities exist. Decisions for recovery of these materials should be made if the recovery capacity is not a problem. 


\section{CONCLUSIONS}

This evaluation method is a useful technique for ranking special case wastes to determine the priorities that should be used for implementing treatment methods that will enable the special case waste to be disposed. The ranking also shows which items should continue to be safely stored. The evaluation presented in this report can be improved by forming a technical evaluation group of recognized experts from the various field offices in the DOE complex to perform the evaluation. However, the evaluation presented in this report should be reviewed by cognizant DOE-HQ personnel or others selected by them to determine if improvement is warranted. For example, availability of Treatment and Storage facilities and whether they comply with regulations are always subject to change. Therefore, any evaluation made would soon have some descrepancies. The evaluation in this report is useful now and any descrepancies that are found can be easily corrected.

The full spreadsheet (Lotus 1-2-3) ranking of all 279 special case waste data record sheets is available on diskette, but was too large to easily include as an appendix to this report. The summary Tables (27 and 28) provide abbreviated information on the items at both ends of the priority evaluation ranking, but do not include the majority of the items in the middle of the spreadsheet. In addition all of the data in Tables 16 through 25 are also available on diskette as Lotus spreadsheets. The priority evaluation Tables (16B through 25B) include formulas that automatically calculate the priority rating and the priority evaluation when the numbers are changed. These spreadsheets can therefore be easily revised and updated to reflect any changes that may occur. 
APPENDIX A

SPECIAL CASE WASTE TREATMENT AND DISPOSAL STRATEGY DECISION TREE

A-1 


\section{SPECIAL CASE WASTE TREATMENT AND DISPOSAL STRATEGY \\ DECISION TREE}

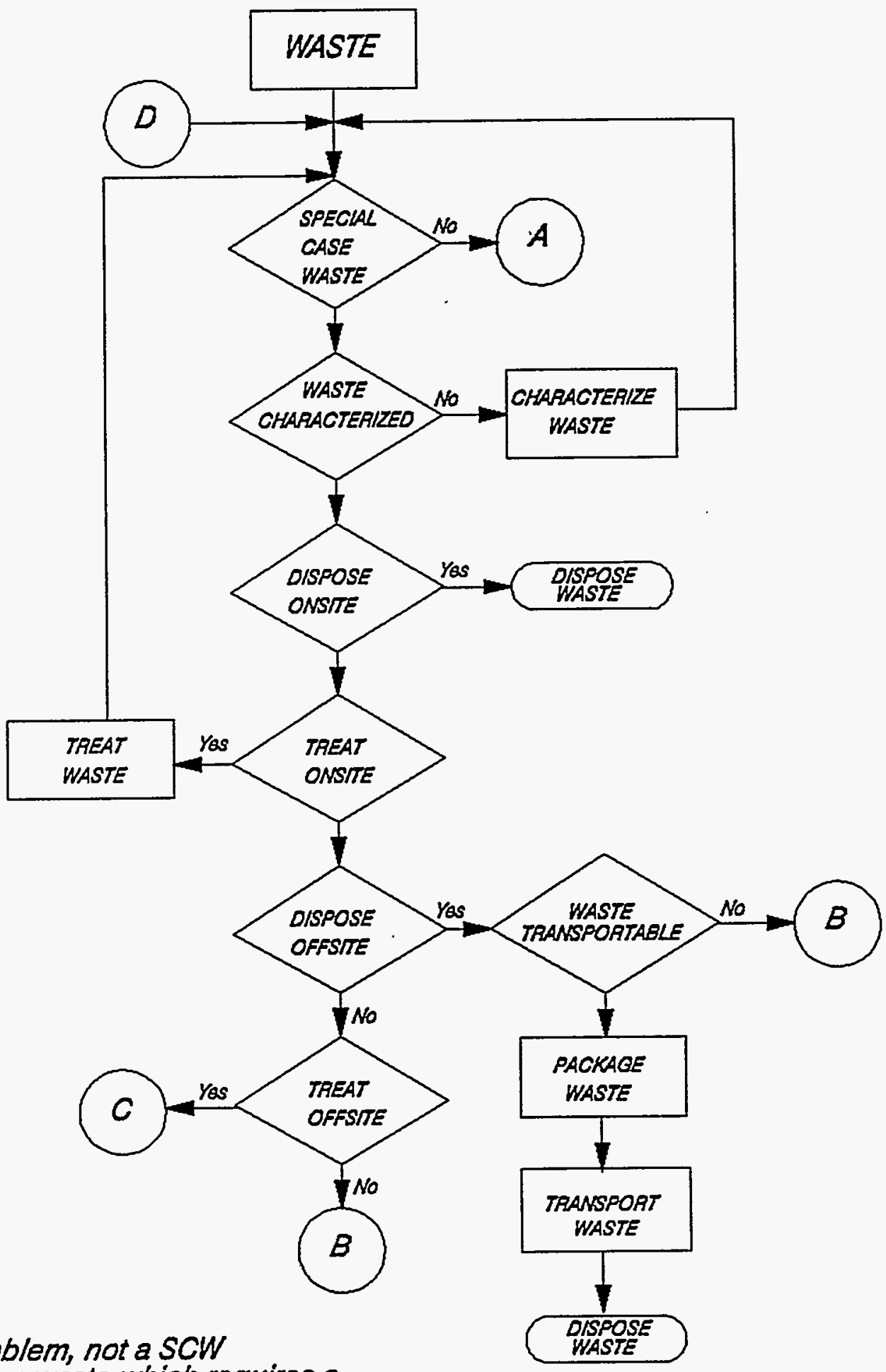

A. No problem, not a SCW

$B$. Problem waste which requires a

Priority Evaluation

C. Continuation to next page

D. Continuation from next page

ATTACHMENT NO. 1 
SPECIAL CASE WASTE TREATMENT, STORAGEAND DISPOSAL STRATEGY

\section{DECISION TREE}

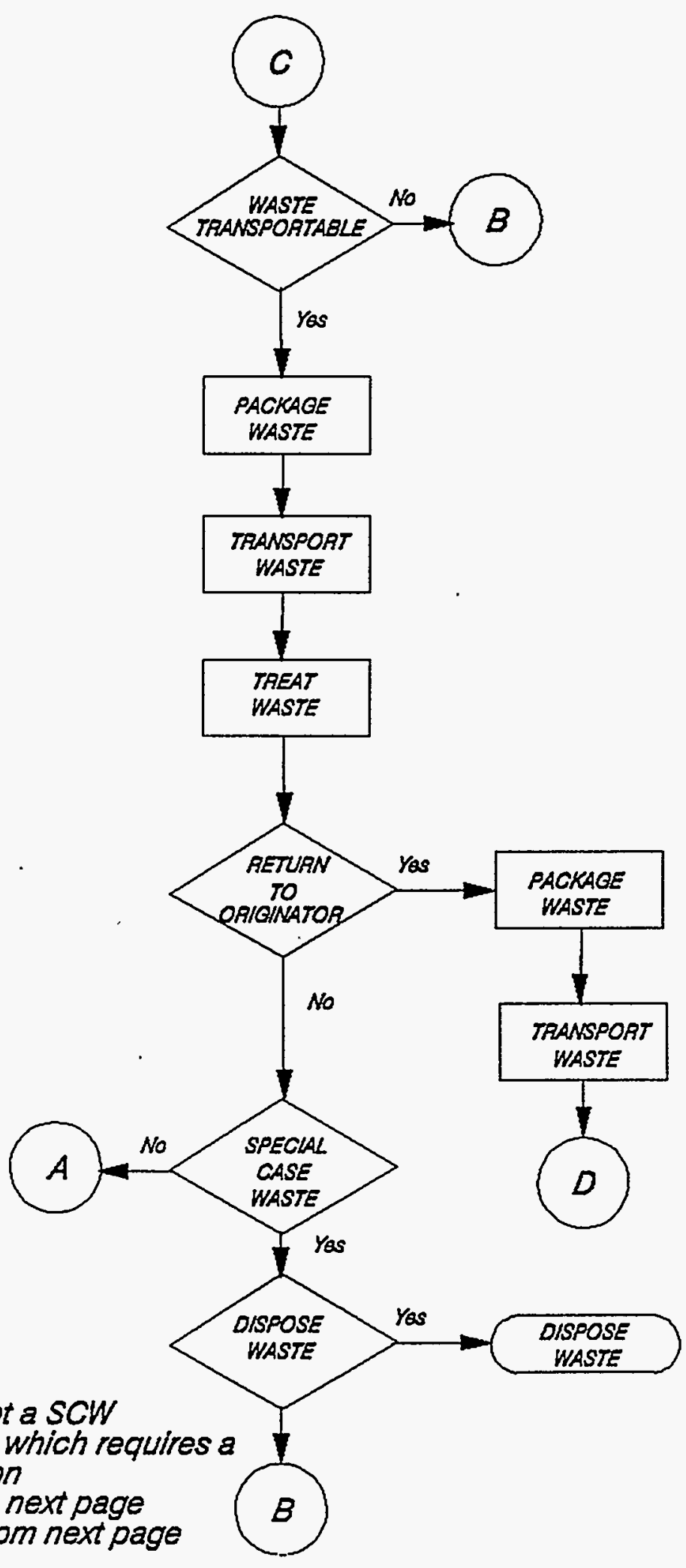

A. No problem, not a SCW

B. Problem Waste which requires a Priority Evaluation

C. Continuation to next page

D. Continuation from next page 